\title{
18. MINERALOGY OF SEDIMENTS CORED DURING DEEP SEA DRILLING PROJECT LEGS 58-60 IN THE NORTH AND SOUTH PHILIPPINE SEA: RESULTS OF X-RAY DIFFRACTION ANALYSES ${ }^{1}$
}

\author{
Ulrich Nagel, German Müller, and Dieter Schumann, Institut für Sedimentforschung der Universität \\ Heidelberg, Heidelberg D-6900, Federal Republic of Germany
}

\begin{abstract}
Sediments recovered by drilling during Legs 58,59 , and 60 in the North and South Philippine Sea have been analyzed by X-ray diffractometry. The $\mathrm{CaCO}_{3}$ content was measured separately. The sites encompass several volcanic ridges and intervening inter-arc basin troughs as well as sites on the Mariana arc fore-arc sediment prism and the Mariana Trench. The sediments at all sites received major volcanogenic input from the various arcs; they tend to be rich in volcanic glass, with associated quartz, feldspar, pyroxenes and amphibole. Carbonate is a major component only at Site 445 at the southern end of the Daito Ridge, and at Site 448 on the Palau-Kyushu Ridge. All other sites were either deep relative to the carbonate compensation depth or had very high non-carbonate sedimentation rates. Clay minerals are mainly smectite and illite with lesser variable proportions of chlorite and kaolinite. Smectite predominates over illite except at sites in the Shikoku Basin and the Daito Ridge, and at one site in the Mariana Trench. At several sites, smectite increases and illite decreases with depth. Principal zeolites are phillipsite and clinoptilolite. Analcime occurs in some samples.
\end{abstract}

\section{INTRODUCTION}

This chapter reports the results of X-ray diffraction analyses of sediments recovered from Deep Sea Drilling Project Legs 58, 59, and 60. Site locations are shown on Figure 1. Leg 58 drilling included three sites-Sites 442 through 444-in the Shikoku Basin, and two sitesSites 445 and 446-in the Daito Ridge and Basin Province. Legs 59 and 60 were primarily an east-west transect with holes drilled on successively younger ridges and back-arc spreading troughs. From west to east, the ridges are the Palau-Kyushu Ridge (Site 445), the West Mariana Ridge (Site 451), and the Mariana arc (Sites 458 and 459). The basins are the West Philippine Sea (Site 447), the Parece-Vela Basin (Sites 449 and 450), and the actively spreading Mariana Trough (Sites 454, 455 , and 456). Two additional sites-Sites 460 and 461were drilled in the Mariana Trench and one on the Pacific plate in the Mariana Basin (Site 452). This report lists data from all these sites but Sites 452, 455, and 457 .

\section{METHODS}

X-ray diffraction procedures as described by Mann and Müller (1980) have been employed. Carbonate contents were determined by the "Karbonat-Bombe" method of Müller and Gastner (1971).

\section{RESULTS}

X-ray diffraction results are listed in Table 1 (Leg 58 ), Table 2 (Leg 59), and Table 3 (Leg 60). The data are plotted versus depth and summary lithologies on Figures 1-5 (Leg 58), Figures 6-9 (Leg 59), and Figure 10

\footnotetext{
1 Initial Reports of the Deep Sea Drilling Project, Volume 60.
}

(Leg 60). A brief synopsis of the results from each site is given in the following paragraphs.

\section{LEG 58}

\section{Site 442 (see Fig. 2)}

The Quaternary period is characterized by a dark greenish gray mud and a clay sequence with scattered ash zones. Clay minerals (and amorphous components) represent about 60 percent of the bulk mineralogy. Quartz is the most abundant mineral of the non-clay fraction $(\sim 20 \%)$, and three specimens (Samples 442A2-2, 103-105 cm, 442A-4-2, 84-86 cm, and 442A-15-2, $16-18 \mathrm{~cm}$ ) contain calcite in amounts ranging between 3 and 5 percent. The lowest part of the Quaternary yields decreasing illite contents (from $\sim 60$ to $34 \%$ ), whereas smectite rises from $\sim 27$ to 53 percent. Kaolinite and chlorite remain almost constant. Pliocene samples contain $\sim 70$ percent clay minerals (and amorphous material). Again the $>2-\mu \mathrm{m}$ fraction is dominated by quartz $(\sim 20 \%)$. No differences between samples of the yellow and dark brown mud have been observed.

Clay minerals become more abundant in Miocene samples (from $\sim 70$ to $\sim 80 \%$ ), whereas quartz decreases to $<5$ percent. Feldspar has been found throughout Hole $442 \mathrm{~A}$, but its content does not vary as much as that of quartz; all samples contain at least 2 percent and the maximum is 12 percent (Sample 30-2, 24-26 cm). Sample $29-2,23-25 \mathrm{~cm}$ of an interbedded ash and clay sequence is light gray in color and has 2.5 percent feldspar and 2 percent quartz; its main components $(\sim 95 \%)$ are volcanic ash and clay minerals with smectite, which comprises $\sim 81$ percent of the $<2-\mu \mathrm{m}$ fraction. The early Miocene, dark brown zeolithic clay of Unit IV contains minor amounts of quartz $(\sim 4 \%)$ in addition to feldspar, smectite, and amorphous material. 
Table 1. Summary of X-ray mineralogy, samples, sample depths, and X-ray diffraction results of Sites 442-446, Leg 58.

\begin{tabular}{|c|c|c|c|c|c|c|c|c|c|c|c|c|c|c|c|c|}
\hline & & & & & & Bul & Mineral & ogy $=100$ & & & & & & y Miner & alogy $=1$ & $0 \%$ \\
\hline $\begin{array}{l}\text { Core-Section } \\
\text { (interval } \\
\text { in } \mathrm{cm} \text { ) }\end{array}$ & Sample & $\begin{array}{l}\text { Depth } \\
(\mathrm{m})\end{array}$ & $\begin{array}{c}\text { and } \\
\text { Volcanic } \\
\text { Glass }\end{array}$ & Quartz & $\begin{array}{l}\text { Feld- } \\
\text { spar }\end{array}$ & Pyroxene & $\begin{array}{l}\text { Horn- } \\
\text { blende }\end{array}$ & $\begin{array}{l}\text { Carbon- } \\
\text { ates }\end{array}$ & $\begin{array}{l}\text { Opal- } \\
\text { CT }\end{array}$ & $\begin{array}{l}\text { Clinop- } \\
\text { tilolite }\end{array}$ & $\begin{array}{l}\text { Anal- } \\
\text { cime }\end{array}$ & $\begin{array}{l}\text { Cristo- } \\
\text { balite }\end{array}$ & Smectite & Illite & Chlorite & Kaolinite \\
\hline Hole $442 \mathrm{~A}$ & & & & & & & & & & & & & & & & \\
\hline $2-2,103-105$ & 1 & 12.04 & 61.4 & 24.7 & 8.8 & Tr. & - & 5.1 & - & - & - & - & 18.0 & 59.0 & 19.0 & 4.0 \\
\hline $3-2,60-62$ & 2 & - & - & - & - & - & - & - & - & - & - & - & - & - & - & - \\
\hline $4-2,84-86$ & 3 & 30.85 & 61.9 & 26.4 & 6.6 & Tr. & - & 5.1 & - & - & - & - & 19.0 & 63.0 & 15.0 & 3.0 \\
\hline $5-2,98-100$ & 4 & - & - & - & - & - & - & - & - & - & - & - & - & - & - & - \\
\hline $6-2,40-42$ & 5 & 49.41 & 61.8 & 26.5 & 6.6 & Tr. & - & Tr. & - & - & - & - & 17.6 & 64.3 & 13.6 & 4.5 \\
\hline $7-2,90-92$ & 6 & - & - & - & - & - & - & - & - & - & - & - & - & - & - & - \\
\hline $8-4,60-62$ & 7 & 71.61 & 66.9 & 27.5 & 5.6 & Tr. & - & Tr. & - & - & - & - & 18.8 & 63.0 & 15.1 & 3.1 \\
\hline $10-2,128-130$ & 8 & 88.29 & - & - & - & - & - & - & - & - & - & - & - & - & - & - \\
\hline $13-3,30-32$ & 9 & 117.30 & 68.7 & 25.3 & 6.0 & Tr. & - & - & - & - & - & - & 26.2 & 57.8 & 14.8 & 1.2 \\
\hline $14-3,54-56$ & 10 & - & - & - & - & - & - & - & - & - & - & - & - & - & - & - \\
\hline $15-2,16-18$ & 11 & 134.67 & 67.5 & 20.9 & 8.6 & Tr. & - & 3.0 & - & - & - & - & 44.9 & 42.9 & 10.5 & 1.2 \\
\hline $16-2,12-14$ & 12 & - & - & - & - & - & - & - & - & - & - & - & $\bar{u}$ & - & - & - \\
\hline $17-2,70-72$ & 13 & 154.21 & 73.6 & 21.4 & 5.0 & Tr. & - & - & - & - & - & - & 34.1 & 52.4 & 12.3 & 1.1 \\
\hline $18-2,125-127$ & 14 & - & - & - & - & - & - & - & - & - & - & - & - & - & - & - \\
\hline $18-3,18-20$ & 15 & 164.69 & 71.9 & 20.1 & 8.0 & Tr. & - & - & - & - & - & - & 19.8 & 65.1 & 13.4 & 1.7 \\
\hline $19-2,40-42$ & 16 & - & - & - & - & - & - & - & - & - & - & - & - & - & - & - \\
\hline $21-2,100-102$ & 17 & 192.51 & 72.2 & 21.8 & 6.0 & Tr. & - & - & - & - & - & - & 26.0 & 60.1 & 10.4 & 3.5 \\
\hline $22-1,126-128$ & 18 & - & - & - & - & - & - & - & - & - & - & - & - & - & - & - \\
\hline $23-3,90-94$ & 19 & 212.90 & 75.6 & 20.4 & 4.0 & Tr. & - & - & - & - & - & - & 22.1 & 62.3 & 8.3 & 7.3 \\
\hline $24-2,118-120$ & 20 & - & - & - & - & - & - & - & - & - & - & - & - & - & - & - \\
\hline $25-2,50-52$ & 21 & 230.01 & 76.9 & 19.5 & 3.6 & - & - & $\mathrm{Tr}$. & - & - & - & - & 29.3 & 59.5 & 6.9 & 4.3 \\
\hline $26-2,110-112$ & 22 & - & - & - & - & - & - & - & - & - & - & - & - & - & - & - \\
\hline $27-2,120-122$ & 23 & 249.71 & 83.6 & 10.4 & 6.0 & - & - & Tr. & - & - & - & - & 40.6 & 43.7 & 7.8 & 7.8 \\
\hline $28-2,35-37$ & 24 & - & - & - & - & - & - & - & - & - & - & - & - & - & - & - \\
\hline $29-2,23-25$ & 25 & 267.74 & 95 & 2.5 & 2.5 & - & - & - & - & - & - & - & 81.8 & 18.2 & - & - \\
\hline $29-2,124-126$ & 26 & - & - & - & - & - & - & - & - & - & - & - & - & - & - & - \\
\hline $30-2,74-76$ & 27 & 277.75 & 81.2 & 7.8 & 12 & Tr. & - & - & - & - & - & - & 73.2 & 18.4 & 4.6 & 3.8 \\
\hline $31-1,26-28$ & 28 & - & - & - & - & - & - & - & - & - & - & - & - & - & - & - \\
\hline Hole 442B & & & & & & & & & & & & & & & & \\
\hline $1-2,40-42$ & 29 & 271.41 & 85.4 & 8.6 & 8 & - & - & - & - & - & - & - & 66.2 & 25 & 4.4 & 4.4 \\
\hline $1-2,106-108$ & 30 & - & - & - & - & - & - & - & - & - & - & - & - & $\overline{5}$ & $\overline{69}$ & $\bar{s}$ \\
\hline $2-2,11-13$ & 31 & 278.62 & 86.0 & 6.5 & 9 & - & - & - & - & - & - & - & 60.3 & 27.5 & 6.9 & 5.2 \\
\hline $3-2,60-62$ & 32 & - & - & - & - & - & - & - & - & - & - & - & - & - & - & - \\
\hline Hole 443 & & & & & & & & & & & & & & & & \\
\hline $1-2,15-17$ & 1 & 1.66 & 64.5 & 26.0 & 5.0 & - & - & 4.5 & - & - & - & - & 11.0 & 57.1 & 24.9 & 6.9 \\
\hline $2-2,89-91$ & 2 & - & - & - & - & - & - & - & - & - & - & - & - & - & - & - \\
\hline $3-2,63-65$ & 3 & 18.64 & 69.3 & 24.7 & 6.0 & - & - & - & - & - & - & - & 18.0 & 63.1 & 12.6 & 6.3 \\
\hline $4-2,65-67$ & 4 & - & - & - & - & - & - & - & - & - & - & - & $\bar{u}$ & - & - & - \\
\hline $5-2,42-44$ & 5 & 37.43 & 63.0 & 26.0 & 5.2 & - & - & 5.8 & - & - & - & - & 14.4 & 60.3 & 18.1 & 10.6 \\
\hline $6-3,30-32$ & 6 & - & - & - & - & - & - & - & - & - & - & - & - & - & - & - \\
\hline $9-4,80-82$ & 7 & 78.81 & 61.0 & 26.4 & 5.6 & Tr. & - & 7.0 & - & - & - & - & 11.7 & 63.5 & 18.4 & 6.3 \\
\hline $10-2,131-133$ & 8 & - & - & - & - & - & - & - & - & - & - & - & - & - & - & - \\
\hline $11-2,125-127$ & ${ }_{10}^{9}$ & 95.26 & 63.9 & 23.4 & 5.0 & - & - & 7.7 & - & - & - & - & 19.8 & 61.4 & 12.4 & 6.4 \\
\hline $\begin{array}{l}14-5,40-42 \\
15-2,43-45\end{array}$ & $\begin{array}{l}10 \\
11\end{array}$ & 122.94 & 72.5 & 22.1 & 5.4 & Tr. & - & - & - & - & - & - & 18.5 & 63.2 & 12.2 & \\
\hline $\begin{array}{l}15-2,43-45 \\
17-4,67-69\end{array}$ & 12 & - & - & - & - & - & $\overline{-}$ & $\overline{-}$ & $\bar{z}$ & $\overline{-}$ & - & - & 18.9 & ${ }_{-}^{03.2}$ & 12.2 & 6.1 \\
\hline $18-2,131-133$ & 13 & 161.82 & 66.9 & 21.7 & 5.0 & Tr. & - & 6.4 & - & - & - & - & 16.8 & 63.4 & 10.9 & 8.9 \\
\hline $21-2,31-33$ & 14 & - & - & - & - & - & - & - & - & - & - & - & - & - & - & - \\
\hline $22-2,129-131$ & 15 & 199.60 & 73.3 & 10.9 & 4.8 & - & - & 7.0 & - & - & - & - & 33.3 & 49.1 & 7.9 & 9.6 \\
\hline $23-2,30-32$ & 16 & - & - & - & - & - & - & - & - & - & - & - & $\overline{-}$ & - & - & - \\
\hline $24-3,25-27$ & 17 & 219.26 & 68.6 & 18.2 & 6.4 & - & - & 7.0 & - & - & - & - & 17.7 & 64.6 & 7.8 & 9.9 \\
\hline $25-2,100-102$ & 18 & - & - & - & - & $\bar{x}$ & - & - & - & - & - & - & - & - & - & - \\
\hline $26-2,80-82$ & 19 & 237.31 & 70.0 & 18.6 & 5.0 & Tr. & - & 6.4 & - & - & - & - & 27.3 & 50.2 & 13.0 & 9.4 \\
\hline $27-2,72-74$ & 20 & - & - & - & - & - & - & - & - & - & - & - & - & - & - & - \\
\hline $28-2,30-32$ & 21 & 255.81 & 74.2 & 20.8 & 5.0 & Tr. & - & - & - & - & - & - & 21.1 & 58.1 & 9.5 & 11.2 \\
\hline $29-2,104-106$ & 22 & - & - & - & - & - & - & - & - & - & - & - & - & - & - & - \\
\hline $30-2,94-96$ & 23 & 275.45 & 83.0 & 12.4 & 4.6 & $\mathrm{Tr}$. & - & - & - & - & - & - & 21.6 & 61.3 & 8.6 & 8.6 \\
\hline $31-2,69-71$ & 24 & - & - & - & - & - & - & - & - & - & - & - & - & - & $\overline{-}$ & - \\
\hline $32-1,62-64$ & 25 & 292.63 & 78.0 & 13 & 5.2 & Tr. & - & 3.8 & - & - & - & - & 39.6 & 40.9 & 7.8 & 11.7 \\
\hline $33-2,138-140$ & 26 & - & - & - & - & - & - & - & - & - & - & - & - & - & - & - \\
\hline $34-2,65-67$ & 27 & 313.16 & 70.5 & 9.1 & 4.4 & Tr. & - & 16.0 & - & - & - & - & 43.7 & 43.7 & 4.7 & 7.8 \\
\hline $35-2,44-46$ & 28 & - & - & - & - & - & - & - & - & - & - & - & - & - & - & - \\
\hline $36-2,34-36$ & 29 & 331.85 & 69.5 & 6.5 & 4.0 & Tr. & - & 20.5 & - & - & - & - & 50.0 & 37.0 & 4.6 & 8.3 \\
\hline $39-2,32-34$ & 30 & - & - & - & - & - & - & - & - & - & - & - & - & - & - & - \\
\hline $40-2,41-43$ & 31 & 369.92 & 75.6 & 10.4 & 5.0 & Tr. & - & 9.0 & - & - & - & - & 45.9 & 40.0 & 7.1 & 7.1 \\
\hline $42-1,34-36$ & 32 & - & - & - & - & - & - & - & - & - & - & - & - & - & - & - \\
\hline $43-2,110-112$ & 33 & 398.35 & 87.9 & 7.5 & 4.6 & - & - & - & - & - & - & - & 60.9 & 26.1 & 8.7 & 4.3 \\
\hline $45-2,52-54$ & 34 & - & - & - & - & - & - & - & - & - & - & - & - & - & - & - \\
\hline $46-2,61-62$ & 35 & 427.11 & 85.1 & 8.5 & 6.4 & Tr. & - & Tr. & - & - & - & - & 60.7 & 27.7 & 7.5 & 4.0 \\
\hline $47-1,45-47$ & 36 & - & - & - & - & - & - & - & - & - & - & - & - & - & - & - \\
\hline $48-1,143-145$ & 37 & 445.44 & 60.5 & 5.7 & 2.4 & - & - & 31.4 & - & - & - & - & 62.9 & 24.1 & 7.8 & 5.2 \\
\hline $49-1,110-112$ & 38 & - & - & - & - & - & - & - & - & - & - & - & - & - & - & - \\
\hline Hole 444 & & & & & & & & & & & & & & & & \\
\hline $2-2,66-68$ & 1 & 8.17 & 67.5 & 25 & 6 & - & $1-2$ & - & - & - & - & - & 8.3 & 70.5 & 16.6 & 4.8 \\
\hline $3-2,73-75$ & 2 & - & - & - & - & - & - & - & - & - & - & - & - & - & - & - \\
\hline $4-2,35-37$ & 3 & 26.86 & 68.0 & 22.7 & 4 & $1-2$ & - & 3.8 & - & - & - & - & 10.8 & 68.6 & 14.9 & 5.6 \\
\hline $5-2,67-69$ & 4 & - & - & - & - & - & - & - & - & - & - & - & - & - & - & - \\
\hline $7-1,42-42$ & 5 & 53.91 & 73.4 & 21.4 & 5.2 & - & - & Tr. & - & - & - & - & 19.3 & 63.5 & 11.8 & 5.4 \\
\hline $10-2,61-63$ & 6 & - & - & - & - & - & - & - & - & - & - & - & - & - & - & - \\
\hline
\end{tabular}


Table 1. (Continued).

\begin{tabular}{|c|c|c|c|c|c|c|c|c|c|c|c|c|c|c|}
\hline & & & & & & Bull & Mineral & $\log y=10 x$ & & & & & & y Miner \\
\hline $\begin{array}{l}\text { Core-Section } \\
\text { (interval } \\
\text { in } \mathrm{cm} \text { ) }\end{array}$ & Sample & $\begin{array}{l}\text { Depth } \\
\text { (m) }\end{array}$ & $\begin{array}{l}\text { Clay } \\
\text { Minerals } \\
\text { and } \\
\text { Volcanic } \\
\text { Glass }\end{array}$ & Quartz & $\begin{array}{l}\text { Feld- } \\
\text { spar }\end{array}$ & Pyroxene & $\begin{array}{l}\text { Horn- } \\
\text { blende }\end{array}$ & $\begin{array}{l}\text { Carbon- } \\
\text { ates }\end{array}$ & $\begin{array}{l}\text { Opal- } \\
\text { CT }\end{array}$ & $\begin{array}{l}\text { Clinop- } \\
\text { tilolite }\end{array}$ & $\begin{array}{l}\text { Anal- } \\
\text { cime }\end{array}$ & $\begin{array}{c}\text { Cristo- } \\
\text { balite }\end{array}$ & Smectite & Illite \\
\hline Hole 444A & & & & & & & & & & & & & & \\
\hline $1-2,20-22$ & 7 & 83.71 & 61.8 & 11.8 & 4.0 & - & Tr. & 22.4 & - & - & - & - & 19.5 & 63.3 \\
\hline $2-2,100-102$ & 8 & - & - & - & - & - & - & - & - & - & - & - & - & - \\
\hline $3-2,80-82$ & 9 & 103.31 & 76.4 & 13.5 & 6.4 & $1-2$ & $1-2$ & Tr. & - & - & - & - & 28.3 & 56.1 \\
\hline $6-2,110-112$ & 10 & - & - & $\overline{-1}$ & - & - & - & - & - & - & - & - & $\overline{2}$ & - \\
\hline $7-1,50-52$ & 11 & 139.51 & 74.7 & 17.7 & 5.6 & 1 & 1 & $\mathrm{Tr}$. & - & - & - & - & 29.1 & 58.5 \\
\hline $8-1,112-114$ & 12 & - & - & - & - & - & - & - & - & - & - & - & - & - \\
\hline $9-2,70-72$ & 13 & 160.21 & 79.6 & 6.5 & 7.4 & 2 & 2 & 3.2 & - & - & - & - & 47.9 & 40.2 \\
\hline $10-2,68-70$ & 14 & - & - & - & - & - & - & - & - & - & - & - & - & - \\
\hline $11-2,112-114$ & 15 & 179.63 & 84.5 & 4 & 10 & $1-2$ & Tr. & $\mathrm{Tr}$. & - & - & - & - & 50.6 & 35.5 \\
\hline $12-2,75-77$ & 16 & - & - & - & - & - & - & - & - & - & - & - & - & - \\
\hline $13-2,27-29$ & 17 & 197.78 & 78.5 & 6.5 & 7 & $2-3$ & 1 & 4.5 & - & - & - & - & 61.3 & 31.3 \\
\hline $14-2,50-52$ & 18 & - & - & - & - & - & - & - & - & - & - & - & - & - \\
\hline $15-2,48-50$ & 19 & 216.99 & 96.5 & 2.1 & 1.4 & Tr. & Tr. & - & - & - & - & - & 66.3 & 25.6 \\
\hline $16-2,84-86$ & 20 & - & - & - & - & - & - & - & - & - & - & - & - & - \\
\hline $17-1,16-17$ & 21 & 230.16 & 54.9 & 9. & 36 & - & - & - & - & - & - & - & 32.4 & 49.3 \\
\hline $21-2,89-91$ & 22 & - & - & - & - & - & - & - & - & - & - & - & - & - \\
\hline $22-2,52-54$ & 23 & 264.54 & 85.0 & 8.0 & 7 & Tr. & Tr. & - & - & - & - & - & 55.0 & 33.6 \\
\hline $23-1,55-57$ & 24 & - & - & - & - & - & - & - & - & - & - & - & - & - \\
\hline Hole 445 & & & & & & & & & & & & & & \\
\hline $2-2,109-111$ & 1 & 11.10 & 59.7 & 13.0 & 3 & - & - & 44.4 & - & - & - & - & 4.3 & 71.6 \\
\hline $3-2,119-121$ & 2 & - & - & - & - & - & - & - & - & - & - & - & - & - \\
\hline $4-2,87-89$ & 3 & 29.88 & 17.7 & 1.3 & 1 & - & - & 80.0 & - & - & - & - & & 41.1 \\
\hline $5-2,49-51$ & 4 & - & - & - & - & - & - & - & - & - & - & - & - & - \\
\hline $6-2,95-97$ & 5 & 48.96 & 30.9 & 7.1 & 1.6 & - & - & 63.5 & - & - & - & - & 8.7 & 68.5 \\
\hline $7-5,30-32$ & 6 & - & - & - & - & - & - & - & - & - & - & - & - & - \\
\hline $8-4,100-102$ & 7 & 71.1 & 34.4 & 9.1 & 1.6 & - & - & 55.9 & - & - & - & - & 8.4 & 69.3 \\
\hline $9-1,16-18$ & 8 & - & - & - & - & - & - & - & - & - & - & - & - & - \\
\hline $10-4,50-52$ & 9 & 89.51 & 17.7 & 2.6 & 1 & - & - & 78.7 & - & - & - & - & 13.0 & 66.3 \\
\hline $11-3,100-102$ & 10 & - & - & - & - & - & - & - & - & - & - & - & $\overline{-}$ & - \\
\hline $12-2,22-24$ & 11 & 105.23 & 20.9 & 3.0 & 1.2 & - & - & 74.9 & - & - & - & - & 35.6 & 47.4 \\
\hline $13-2,58-60$ & 12 & - & - & - & - & - & - & - & - & - & - & - & - & - \\
\hline $14-1,94-96$ & 13 & 123.45 & 15.1 & 2.6 & 1 & - & - & 81.3 & - & - & - & - & 26.2 & 57.0 \\
\hline $15-2,60-62$ & 14 & - & - & - & - & - & - & - & - & - & - & - & $\bar{n}$ & - \\
\hline $16-2,90-92$ & 15 & 143.9 & 13.1 & 3.4 & 1 & - & - & 82.5 & - & - & - & - & 22.3 & 53.2 \\
\hline $17-2,47-49$ & 16 & - & - & - & - & - & - & - & - & - & - & - & - & - \\
\hline $18-2,64-66$ & 17 & 162.65 & 15.1 & 2.6 & 1 & - & - & 81.3 & - & - & - & - & 29.5 & 56.1 \\
\hline $19-2,89-91$ & 18 & - & - & - & - & - & - & - & - & - & - & - & $\bar{x}$ & - \\
\hline $20-2,43-45$ & 19 & 181.44 & 12.8 & 2.1 & 3.2 & - & - & 81.9 & - & - & - & - & 25.3 & 58.1 \\
\hline $21-2,31-33$ & 20 & - & - & - & - & - & - & - & - & - & - & - & - & - \\
\hline $22-2,57-59$ & 21 & 200.58 & 13.5 & 1.7 & 1 & - & - & 83.8 & - & - & - & - & 34.9 & 45.9 \\
\hline $23-2,109-111$ & 22 & - & - & - & - & - & - & - & - & - & - & - & - & - \\
\hline $24-2,63-65$ & 23 & 219.14 & 22.4 & 2.6 & 1.4 & - & - & 73.6 & - & - & - & - & 19.0 & 59.5 \\
\hline $25-2,97-99$ & 24 & - & - & - & - & - & - & - & - & - & - & - & - & - \\
\hline $26-2,106-108$ & 25 & 239.07 & 23.4 & 3.9 & 1 & - & - & 71.7 & - & - & - & - & 24.6 & 54.6 \\
\hline $27-2,106-108$ & 26 & - & - & - & - & - & - & - & - & - & - & - & $\bar{x}$ & - \\
\hline $28-2,87-89$ & 27 & 257.88 & 27.2 & 2.6 & 1.6 & - & - & 68.6 & - & - & - & - & 45.0 & 34.4 \\
\hline $29-2,79-81$ & 28 & 267.30 & 35.7 & 5.2 & 2 & - & - & 57.1 & - & - & - & - & 33.0 & 41.8 \\
\hline $30-2,69-71$ & 29 & - & - & - & - & - & - & - & - & - & - & - & - & - \\
\hline $31-2,46-48$ & 30 & 285.97 & 36.3 & 5.2 & 1.4 & - & - & 57.1 & - & - & - & - & 43.4 & 40.8 \\
\hline $32-2,60-62$ & 31 & - & - & - & - & - & - & - & - & - & - & - & - & - \\
\hline $33-2,143-145$ & 32 & 305.95 & 25 & 2.9 & 1 & - & - & 71.1 & - & - & - & - & 60.3 & 28.4 \\
\hline $34-2,46-48$ & 33 & 314.47 & 26.6 & 1.3 & 1 & - & - & 71.1 & - & - & - & - & 68.7 & 20.8 \\
\hline $35-2,92-94$ & 34 & - & - & - & - & - & - & - & - & - & - & - & - & - \\
\hline $36-2,31-33$ & 35 & 333.32 & 22.2 & 2.0 & 1 & - & - & 64.8 & - & - & - & - & 60.3 & 24.1 \\
\hline $37-2,90-92$ & 36 & - & - & - & - & - & - & - & - & - & - & - & - & - \\
\hline $38-2,104-106$ & 37 & 353.05 & 16.2 & 1.1 & 1.4 & - & - & 81.3 & - & - & - & - & 83.7 & 9.8 \\
\hline $39-2,91-93$ & 38 & - & - & - & - & - & - & - & - & - & - & - & - & - \\
\hline $40-1,77-79$ & 39 & 371.78 & 14.7 & 1.3 & 1.4 & - & - & 73.6 & - & - & - & - & 100 & - \\
\hline $41-2,48-49$ & 40 & - & - & - & - & - & - & - & - & - & - & - & - & - \\
\hline $42-2,44-45$ & 41 & 390.44 & 13.6 & 1 & 1 & - & - & 84.4 & - & - & - & - & 100 & - \\
\hline $43-2,88-89$ & 42 & - & - & - & - & - & - & - & - & - & - & - & - & - \\
\hline $44-2,124-125$ & 43 & 410.25 & 7.8 & 1 & 1 & - & - & 90.2 & - & - & - & - & 89 & \\
\hline $45-2,123-124$ & 44 & - & - & - & - & - & - & - & - & - & - & - & - & - \\
\hline $46-2,97-98$ & 45 & 428.97 & 8.5 & 1 & 1 & - & - & 89.5 & - & - & - & - & 76.3 & 15.8 \\
\hline $47-2,46-47$ & 46 & - & - & - & - & - & - & - & - & - & - & - & - & - \\
\hline $48-2,107-109$ & 47 & 448.08 & 15.1 & 1 & 2.6 & - & - & 81.3 & - & - & - & - & 100 & - \\
\hline $49-2,110-112$ & 48 & - & - & - & - & - & - & - & - & - & - & - & - & - \\
\hline $50-2,32-34$ & 49 & 466.33 & 14.5 & 1 & 2 & - & - & 82.5 & - & - & - & - & 100 & - \\
\hline $51-2,5-7$ & 50 & - & - & - & - & - & - & - & - & - & - & - & - & - \\
\hline $52-2,18-20$ & 51 & 485.19 & 18.3 & 1 & 2 & - & - & 78.7 & - & - & - & - & 90.0 & 7.6 \\
\hline $53-2,56-58$ & 52 & - & - & - & - & - & - & - & - & - & - & - & - & - \\
\hline $54-2,56-58$ & 53 & 504.57 & 12.4 & - & - & - & - & 87.6 & - & - & - & - & 100 & - \\
\hline $55-2,64-66$ & 54 & 514.15 & 17.9 & 0.7 & 2 & - & - & 79.4 & - & - & - & - & 86.2 & 10.0 \\
\hline $56-2,54-56$ & 55 & 523.55 & 31.9 & 1.3 & 5.0 & Tr. & Tr. & 62.8 & - & - & - & - & 76.8 & 16.5 \\
\hline $57-2,74-76$ & 56 & - & - & - & - & - & - & - & - & - & - & - & - & - \\
\hline $59-2,83-85$ & 57 & 552.34 & 25 & - & 1.6 & - & - & & - & - & - & - & 100 & - \\
\hline $60-2,127-129$ & 58 & - & - & - & - & - & - & - & - & - & - & - & - & - \\
\hline $61-2,109-111$ & 59 & 571.6 & 57.1 & 1 & 5.0 & 1 & 1 & 34.9 & 10 & - & - & - & 100 & - \\
\hline $62-2,44-46$ & 60 & 580.45 & 46.6 & 0.8 & 3 & 1 & - & 47.6 & - & - & - & - & 100 & - \\
\hline $63-2,127-129$ & 61 & - & - & - & - & - & - & - & - & - & - & - & - & - \\
\hline $64-2,18-20$ & 62 & 599.14 & 57.8 & 1.3 & 3.4 & 1 & 1 & 35.5 & 15 & - & - & - & 100 & - \\
\hline
\end{tabular}


Table 1. (Continued).

\begin{tabular}{|c|c|c|c|c|c|c|c|c|c|c|c|c|c|c|c|c|}
\hline & & & & & & Bult & Minera & $\log y=100$ & & & & & & ay Miner & alog $y=1$ & $100 \%$ \\
\hline $\begin{array}{l}\text { Core-Section } \\
\text { (interval } \\
\text { in } \mathrm{cm} \text { ) }\end{array}$ & Sample & $\begin{array}{l}\text { Depth } \\
\text { (m) }\end{array}$ & $\begin{array}{l}\text { Minerals } \\
\text { and } \\
\text { Volcanic } \\
\text { Glass }\end{array}$ & Quartz & $\begin{array}{l}\text { Feld- } \\
\text { spar }\end{array}$ & Pyroxene & $\begin{array}{l}\text { Horn- } \\
\text { blende }\end{array}$ & $\begin{array}{l}\text { Carbon- } \\
\text { ates }\end{array}$ & $\begin{array}{l}\text { Opal- } \\
\text { CT }\end{array}$ & $\begin{array}{l}\text { Clinop- } \\
\text { tilolite }\end{array}$ & $\begin{array}{l}\text { Anal- } \\
\text { cime }\end{array}$ & $\begin{array}{l}\text { Cristo- } \\
\text { balite }\end{array}$ & Smectite & Illite & Chlorite & Kaolinite \\
\hline Hole 445 (Cont & & & & & & & & & & & & & & & & \\
\hline $65-2,42-44$ & 63 & - & - & - & - & - & - & - & - & - & - & - & - & - & - & - \\
\hline $66-2,16-18$ & 64 & 618.17 & 53.7 & 1.3 & 2.4 & 1 & 1 & 40.6 & 13 & - & - & - & 100 & - & - & - \\
\hline $67-1,90-97$ & 65 & - & - & - & - & - & - & - & - & - & - & - & - & - & - & - \\
\hline $68-2,41-43$ & 66 & 637.42 & 52 & 1 & 1 & Tr. & Tr. & 40.0 & - & - & - & 6 & 100 & - & - & - \\
\hline $69-2,100-102$ & 67 & - & - & - & - & - & - & - & - & - & - & - & - & - & - & - \\
\hline $70-2,7-9$ & 68 & 656.08 & 52.7 & 2.6 & 5.0 & - & 7 & 26.7 & - & 6 & - & - & 87.1 & 10.4 & & $2.5^{\mathrm{a}}$ \\
\hline $71-2,6-8$ & 69 & - & - & - & - & - & - & - & - & - & - & - & - & - & - & - \\
\hline $72-2,43-45$ & 70 & 675.44 & 67.2 & 2.6 & 5 & $1-2$ & $1-2$ & 14.6 & - & 7.6 & - & - & 84.0 & 10.5 & 4.0 & 1.5 \\
\hline $73-2,84-86$ & 71 & - & - & - & - & - & - & - & - & - & - & - & - & - & - & - \\
\hline $74-2,46-48$ & 72 & 694.47 & 62.4 & 2.0 & 4.6 & Tr. & $1-2$ & 24.1 & - & 5.4 & - & - & 74.2 & 20.9 & 3.7 & 1.2 \\
\hline $75-2,39-41$ & 73 & - & - & - & - & - & - & - & - & - & - & - & - & - & - & - \\
\hline $76-2,93-95$ & 74 & 713.94 & 65 & 4.5 & 2.4 & $?$ & - & 24.1 & - & 5.0 & - & - & 85.6 & 4.8 & 4.8 & 4.8 \\
\hline $77-2,38-40$ & 75 & - & - & - & - & - & - & - & - & - & - & - & - & - & - & - \\
\hline $78-2,60-62$ & 76 & 732.61 & 68.1 & 3.6 & 2.8 & Tr. & Tr. & 35.5 & - & - & - & - & 100 & - & - & - \\
\hline $79-2,52-54$ & 77 & - & - & - & - & - & - & - & - & - & - & - & - & - & - & - \\
\hline $80-2,138-140$ & 78 & 751.89 & 63 & 2.2 & 5.4 & Tr. & $1-2$ & 27.9 & - & - & - & - & 100 & - & - & - \\
\hline $81-2,56-58$ & 79 & - & - & - & - & $\because$ & - & - & - & - & - & - & - & - & - & - \\
\hline $82-2,70-72$ & 80 & 770.71 & 82.2 & 2.6 & 4.6 & $1-2$ & $1-2$ & 7.6 & - & - & - & - & 89.6 & - & - & 10.4 \\
\hline $83-2,76-78$ & 81 & - & - & - & - & - & - & - & - & - & - & - & - & - & - & - \\
\hline $84-2,93-95$ & 82 & 791.94 & 78.4 & 3.6 & 2.8 & - & - & 15.2 & - & - & Tr. & - & 83.5 & - & - & 16.5 \\
\hline $85-2,95-97$ & 83 & - & - & - & - & - & - & - & - & - & - & - & - & - & - & - \\
\hline $86-2,56-58$ & 84 & 808.57 & 77.4 & 3.6 & 5.4 & $\mathrm{Tr}$. & Tr. & 7.0 & - & - & 6.6 & - & 88.2 & - & - & 11.8 \\
\hline $87-2,40-42$ & 85 & - & - & - & - & - & - & - & - & - & - & - & - & - & - & - \\
\hline $88-2,58-60$ & 86 & 827.59 & 79.5 & 1.7 & 10 & 1 & $1-2$ & 6.3 & - & - & - & - & 87.6 & - & - & 12.4 \\
\hline $89-3,75-77$ & 87 & - & - & - & - & - & - & - & - & - & - & - & - & - & - & - \\
\hline $90-2,70-72$ & 88 & 846.71 & 73.2 & 3.2 & 4.6 & - & - & 11.4 & - & 2 & 5.6 & - & 85.1 & - & - & 14.9 \\
\hline $91-2,50-52$ & 89 & - & - & - & - & - & - & - & - & - & - & - & - & - & - & - \\
\hline $92-2,65-67$ & 90 & 865.66 & 65.5 & 3.4 & 16 & - & - & 5.1 & - & - & 10 & - & 84.7 & - & - & 15.3 \\
\hline $93-2, \quad 146-148$ & 91 & - & - & - & - & - & - & - & - & - & - & - & - & - & - & - \\
\hline $94-2,96-98$ & 92 & 884.97 & 69 & 7.3 & 13 & - & - & $3-4$ & - & - & 7 & - & 81.8 & - & - & 18.2 \\
\hline Hole 446 & & & & & & & & & & & & & & & & \\
\hline $1-1,99-101$ & 1 & 1.0 & 67 & 26 & 5 & -1 & 1 & - & - & - & - & - & 14.1 & 64.6 & 21.3 & - \\
\hline $3-3,120-122$ & 2 & - & - & - & - & - & - & - & - & - & - & - & - & - & - & - \\
\hline $5-4,94-96$ & 3 & 36.95 & 71.5 & 17.5 & 9.0 & -1 & 1 & - & - & - & - & - & 33.1 & 47.5 & 11.0 & 8.3 \\
\hline $6-5,42-44$ & 4 & - & - & - & - & - & - & - & - & - & - & - & - & - & - & - \\
\hline $7-2,91-93$ & 5 & 51.42 & 78.7 & 11.3 & 7.0 & 1 & 2 & - & - & - & - & - & 57.1 & 28.4 & 7.5 & 6.9 \\
\hline $8-2,76-78$ & 6 & - & - & - & - & - & - & - & - & - & - & - & - & - & - & - \\
\hline $9-2,97-99$ & 7 & 67.48 & 80.5 & 6.5 & 3.0 & - & - & - & - & - & - & - & 80.6 & 15.5 & - & 3.9 \\
\hline $10-2,108-110$ & $\begin{array}{l}8 \\
9\end{array}$ & - & - & - & - & - & - & - & - & - & - & - & - & - & - & - \\
\hline $11-2,66-68$ & 10 & - & - & - & - & - & - & - & - & - & - & - & - & - & - & - \\
\hline $12-2,105-107$ & 11 & 99.60 & 92.6 & 6.2 & 1.2 & - & - & - & - & - & - & - & 80.1 & 16.5 & 2.7 & 2.7 \\
\hline $13-2,32-34$ & 12 & - & - & - & - & - & - & - & - & - & - & - & - & - & - & - \\
\hline $14-2,61-63$ & 13 & 117.62 & 84.7 & 11.7 & 2.6 & - & - & - & - & - & - & - & 72.2 & 20.4 & 2.8 & 4.5 \\
\hline $15-2,123-125$ & 14 & - & - & - & - & - & - & - & - & - & - & - & - & - & - & - \\
\hline $16-2,38-40$ & is & 136.39 & 95.6 & 2.0 & 2.4 & Tr. & - & - & - & - & - & - & 97.5 & 1.5 & - & 1.0 \\
\hline $18-1,106-108$ & 16 & - & - & - & - & - & - & - & - & - & - & - & - & - & - & - \\
\hline $19-1,147-149$ & 17 & 163.48 & 79.0 & 1.3 & 6 & $1-2$ & $1-2$ & - & - & 11 & - & - & 89.0 & 3.9 & 3.9 & 3.2 \\
\hline $20-1,60-62$ & 18 & - & - & - & - & - & - & - & - & - & - & - & - & - & - & - \\
\hline $21-1,88-90$ & 19 & 182.89 & 89.2 & 2.0 & 3.0 & 1 & $3-4$ & - & - & 1.8 & - & - & 92.2 & - & - & 7.8 \\
\hline $23-1,55-57$ & 20 & - & - & - & - & - & - & - & - & - & - & - & - & - & - & - \\
\hline $24-3,126-129$ & 21 & 214.77 & 79.0 & 3.2 & 9 & $2-3$ & 3 & 3.2 & - & - & - & - & 92.4 & - & - & 7.6 \\
\hline $25-2,27-29$ & 22 & - & - & - & - & - & - & - & - & - & - & - & - & - & - & - \\
\hline $26-2,109-111$ & 23 & 232.10 & 90.0 & - & 2 & Tr. & Tr. & - & - & - & 7 & - & 100 & - & - & - \\
\hline $27-1,142-144$ & 24 & - & - & - & - & - & - & - & - & - & - & - & - & - & - & - \\
\hline $28-1,25-27$ & 25 & 248.76 & 79.7 & 5.8 & 7.0 & 1.2 & 6 & - & - & - & - & - & 93.9 & - & - & 6.1 \\
\hline $29-2,47-49$ & 26 & - & - & - & - & - & - & - & - & - & - & - & - & - & - & - \\
\hline $30-2,126-128$ & 27 & 272.77 & 82.0 & 1.3 & 5.0 & - & 2 & - & - & - & 7.2 & - & 100 & - & - & - \\
\hline $31-2,44-46$ & 28 & - & - & - & - & - & - & - & - & - & - & - & - & - & - & - \\
\hline $32-2,98-100$ & 29 & 288.99 & 71.2 & 1.3 & 3 & $2-3$ & - & - & - & 13 & 9 & - & 100 & - & - & - \\
\hline $33-1,126-128$ & 30 & - & - & - & - & - & - & - & - & - & - & - & - & - & - & - \\
\hline $34-1,35-37$ & 31 & 305.86 & 85.4 & 0.65 & 1 & 2 & - & - & - & - & 13 & - & 100 & - & - & - \\
\hline $36-1,142-144$ & 32 & - & - & - & - & - & - & - & - & - & - & - & - & - & - & - \\
\hline $37-1,125-126$ & 33 & 335.25 & 85.4 & 2.6 & 3.0 & 2 & - & - & - & 7 & - & - & 100 & - & - & - \\
\hline $38-1,12-14$ & 34 & - & - & - & - & - & - & - & - & - & - & - & - & - & - & - \\
\hline $39-1,26-28$ & 35 & 353.27 & 62.9 & 10.4 & 2.6 & 2 & 2 & 14.1 & - & 6 & - & - & 73.7 & - & 13.1 & 13.1 \\
\hline $40-2,37-38$ & 36 & - & - & - & - & - & - & - & - & - & - & - & - & - & - & - \\
\hline $41-2,17-19$ & 37 & 373.18 & 92.0 & 1.8 & 3 & $\operatorname{Tr}$. & 1 & - & - & 3.2 & - & - & 100 & - & - & - \\
\hline $43-2,97-99$ & 38 & 393.48 & - & - & - & - & - & - & - & - & - & - & - & - & - & - \\
\hline Hole 446A & & & & & & & & & & & & & & & & \\
\hline $10-4,60-62$ & $39 a$ & 453.12 & 85.3 & - & 5.0 & - & - & - & - & 7.2 & - & - & 100 & - & - & - \\
\hline $12-3,101-103$ & 39 & 471.02 & 90.0 & 0.6 & 3.2 & Tr. & 1 & - & - & 5.0 & - & - & 100 & - & - & - \\
\hline $13-3,73-75$ & 40 & - & - & - & - & - & - & - & - & - & - & - & - & - & - & - \\
\hline $16-3,110-112$ & 41 & 509.11 & 82.1 & 1.3 & 2 & - & - & - & - & 4.6 & - & - & 100 & - & - & - \\
\hline $17-2,4-6$ & 42 & - & - & - & - & - & - & - & - & - & - & - & - & - & - & - \\
\hline $18-1,55-57$ & 43 & 524.56 & 84.1 & 2.3 & 4 & - & - & - & 6.4 & 3.2 & - & - & 100 & - & - & - \\
\hline $22-1,104-106$ & 44 & - & - & - & - & 1 & Tr. & - & - & - & - & - & - & - & - & - \\
\hline $24-2,23-24$ & 45 & 582.73 & 50.7 & 35.8 & 10 & $1-2$ & 2.5 & - & - & - & - & - & 100 & - & - & - \\
\hline $26-3,59-61$ & 46 & - & - & - & - & - & - & - & - & - & - & - & - & - & - & - \\
\hline $28-1,132-134$ & 47 & 620.33 & 88.5 & - & - & - & - & - & 11.5 & - & - & - & 100 & - & - & - \\
\hline
\end{tabular}

${ }^{\text {a }}$ Not separated due to smallness of amounts. 
Clinoptilolite may be present. The $<2-\mu \mathrm{m}$ fraction is dominated by smectite and contains minor amounts of illite and chlorite/kaolinite.

\section{Site 443 (see Fig. 3)}

The Quaternary section of this site, consisting of dark greenish mud and ash to dark greenish gray nannofossil mud and clayey nannofossil ooze, contains $62-70$ percent clay minerals (including amorphous material). A slight increase of illite and smectite corresponds to decreasing chlorite and kaolinite contents between 2 and 19 meters. The same is true for samples between 78 and 94.5 meters. Relative proportions of the clay minerals remain almost constant. Quartz contents vary from 26 to 22 percent, and feldspar remains constant (5-6\%).

The late and middle Pleistocene dark greenish gray mud of Units IA and IB comprise calcite as well, in amounts ranging from about 5 to 7 percent. Unit IIAPliocene ashy clay and mud-consists of $\sim 70$ percent clay minerals, 0-6.4 percent $\mathrm{CaCO}_{3}, 5-6$ percent feldspar, and $\sim 20$ percent quartz. Illite dominates the $<2$ $\mu \mathrm{m}$ fraction (contents of up to $64 \%$ ), and the illite:smectite ratio is $\sim 4: 1$. Unit IIB (early Pliocene gray clay and mud) contains smaller amounts of quartz-only 10 percent-and illite $(49 \%)$, whereas smectite contents double from $\sim 17$ to 33 percent, carbonate and feldspar remaining constant.

The mineralogy of the late Miocene, dark grayish brown, interbedded clayey nannofossil ooze and mud of Unit III does not differ greatly from that of Unit IIB except for an increase in illite, which corresponds to a lower smectite content (Sample 443-24-3, 25-27 cm); quartz rises from 10.8 to 18 percent. A decreasing illite and increasing smectite content occurs for Sample 443$26-2,80-81 \mathrm{~cm}$. Carbonate is present in the uppermost part of Unit III, but could not be detected in the sample from the lowest part. Also the upper part of the late Miocene olive-gray mudstone (Unit IVA) is carbonatefree. Clay minerals increase from 74 to 83 percent (of which $60 \%$ is illite and $20 \%$ smectite); quartz decreases from 21 to 12 percent.

The following sample (Sample 443-62-1, 62-64 cm) of Unit IVA contains lesser amounts of illite, whereas smectite doubles from $\sim 20$ to $\sim 40$ percent, and $\mathrm{CaCO}_{3}$ from 4 to 16 percent. The sample from the middle Miocene, interbedded nannofossil chalk and mudstone sequence (Unit IVB) contains 21 percent $\mathrm{CaCO}_{3}$, in addition to 6 percent quartz, 14 percent feldspar, and 69 percent clay minerals.

Unit V (middle Miocene) comprises dark greenish gray, interbedded claystone, nannofossil, and calcareous chalk and ash. The four $\mathrm{X}$-rayed samples contain varying amounts $(0-31.4 \%) \mathrm{CaCO}_{3}$, which reflects the aforementioned lithology. The sample with high amounts of $\mathrm{CaCO}_{3}$ (Sample 443-48-1, 143-145 cm) has the least clay content. Chlorite gradually decreases from 25 percent (of the $<2-\mu \mathrm{m}$ fraction) during the upper Pleistocene to 4.5 percent, whereas kaolinite remains almost constant.

\section{Site 444 (see Fig. 4)}

At this site, the third of Leg 58 drilled in the Shikoku Basin, hemipelagic sediments similar to those found at Sites 442 and 443 were again encountered. The sequence begins with 28.6 meters of the late Pleistocene, showing dark greenish gray mud, ash, and calcareous mud (Unit IA), which is underlain by 24.2 meters of early Pleistocene olive and olive-brown, partly vitric mud, ash, and calcareous mud (Unit IB). Unit II (Pliocene) consists of olive-brown, again partly vitric mud, and ash.

Units I and II yielded similar mineralogical compositions with quartz being the dominant bulk sample mineral $(\sim 20 \%)$, followed by feldspar. The $<2-\mu \mathrm{m}$ fraction is composed of illite (the main constituent), smectite, chlorite, and kaolinite. Illite and chlorite together comprise about 75 to 87 percent of the clay minerals present.

Units IIIA and IIIB of late Miocene age consist of brown mud, ash, vitric mud, nannofossil mud, and siliceous mud. Correspondingly, we found in one sample (Sample 444A-1-2, 20-22 cm) 22.4 percent $\mathrm{CaCO}_{3}$, but the other samples were free of carbonate. The nonbiogenic mineralogy is similar to that described above. The next lowest level is a middle Miocene sequence of yellow-brown, olive, and gray mud, ash, nannofossil mud, and siliceous mud (Units IVA and IVB).

In Units I through IVB we note a decrease of quartz, illite, and chlorite, whereas smectite increases and kaolinite remains constant. Also the percentage of (combined) clay minerals and volcanic glass is higher in Units II, III, and IVB than in I. Two samples of Unit IVC-middle Miocene claystone and mudstone-yielded quite different mineralogies, whereby Sample 444A$17-1,16-17 \mathrm{~cm}$ has a high feldspar content $(36 \%)$ and Sample 444A-22-2, 52-54 cm is mainly composed of clay minerals and volcanic glass.

\section{Site 445 (see Fig. 5)}

This site was drilled in a small basin on the southern end of the Daito Ridge in the northwestern Philippean Sea. Unit I (early Pliocene to late Pleistocene or Holocene) is further subdivided into sub-units-IA and IB. Unit IA is a Quaternary to late Pliocene, gray or pale brown clayey foraminifer nannofossil ooze, of which three samples were $\mathrm{X}$-rayed. They vary in $\mathrm{CaCO}_{3}$ between 4 and 80 percent; the sample with the highest $\mathrm{CaCO}_{3}$ has the least quartz and illite contents $(\sim 1$ and $\sim 50 \%$, respectively). In Unit IB-light gray, foraminifer-nannofossil ooze to clayey nannofossil oozecalcite is the main component. Quartz and feldspar comprise only 5 percent (maximum), and smectite generally comprises less than 20 percent of the $<2-\mu \mathrm{m}$ fraction; one sample, however, yielded 35 percent.

Unit IIA-late Miocene to middle Miocene, white, very pale brown to yellowish brown, clayey nannofossil chalk-consists of $\mathrm{CaCO}_{3}$ as its main component and has minor admixtures of quartz, feldspar, and clay minerals. The maximum calcite content is determined to 
Table 2. Summary of X-ray mineralogy, samples, sample depths, and X-ray diffraction results of Sites $447,448,449$, and 451 , Leg 59.

\begin{tabular}{|c|c|c|c|c|c|c|c|c|c|c|c|c|c|}
\hline & & & & & & Bulk Mineral & $g y=11$ & & & & lay Minera & alogy $=10$ & \\
\hline $\begin{array}{l}\text { Core-Section } \\
\text { (interval in } \mathrm{cm} \text { ) }\end{array}$ & Sample & $\begin{array}{l}\text { Depth } \\
\text { (m) }\end{array}$ & $\begin{array}{l}\text { + Volanic } \\
\text { Glass }\end{array}$ & Quar17 & $\begin{array}{l}\text { Feld- } \\
\text { spar }\end{array}$ & Pyroxene & $\begin{array}{l}\text { Horn- } \\
\text { blende }\end{array}$ & $\begin{array}{l}\text { Carbon- } \\
\text { ate }\end{array}$ & Phillipsite & Smectite & Illite & Chlorite & Kaolinite \\
\hline Hole 450 (Cont. & & & & & & & & & & & & & \\
\hline $17-3,24-26$ & 16 & - & - & - & - & - & - & - & - & - & - & - & - \\
\hline $18-1,26-28$ & 17 & 159.77 & 84.7 & -1 & 5.4 & $1-2$ & -1 & 6.4 & - & 100 & - & - & - \\
\hline $19-6,14-17$ & 18 & - & - & - & - & - & - & - & - & - & - & - & - \\
\hline $20-2,13-15$ & 19 & 180.14 & 85.5 & -1 & 5.4 & $1-2$ & $1-2$ & 5.1 & - & 100 & - & - & - \\
\hline $20-3,14-16$ & 20 & - & - & - & - & - & - & - & - & - & - & - & - \\
\hline $21-4,51-53$ & 21 & 193.02 & 88.6 & - & 3.0 & 1 & 1 & 6.4 & - & 100 & - & - & - \\
\hline $22-6,6-8$ & 22 & - & - & - & - & - & - & - & - & - & - & - & - \\
\hline $24-3,10-12$ & 23 & 219.61 & 81.1 & 1.3 & 10.0 & $1-2$ & 1 & 5.1 & - & 100 & - & - & - \\
\hline $26-2,41-43$ & 24 & 267.51 & $8 \overline{27}$ & $\overline{13}$ & $\overline{6}_{60}$ & $\overline{1-2}$ & $\overline{1-2}$ & $\overline{7.0}$ & - & $\overline{0}$ & - & - & - \\
\hline $29-3,49-52$ & 25 & 267.51 & $\begin{array}{l}82,7 \\
-\end{array}$ & 1.3 & 6.0 & -2 & - & 7.0 & - & 100 & - & - & - \\
\hline $30-3,33-35$ & 26 & & 840 & - & $\bar{s}$ & - & 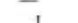 & $\overline{10}$ & - & $-\overline{-}$ & - & - & - \\
\hline $32-3,84-87$ & 27 & 296.36 & 84.0 & -1 & 5.6 & 1 & 1 & 10.2 & - & 100 & - & - & - \\
\hline $33-1,123-124$ & 28 & & $-\overline{2}$ & - & - & - & - & - & - & - & - & - & - \\
\hline $35-3,83-85$ & 29 & 324.84 & 95.2 & - & -1 & - & - & 3.2 & - & 100 & - & - & - \\
\hline $36-1,7-10$ & 30 & - & - & - & - & - & - & - & - & - & - & - & - \\
\hline $36-1,148-150$ & 31 & 331.99 & 81.7 & 2.1 & 3.4 & - & - & 12.8 & - & 100 & - & - & - \\
\hline $36-2,118-122$ & 32 & - & - & - & - & - & - & - & - & - & - & - & - \\
\hline & & & & & & Sulk Mineral & $g y=10$ & & & & & & \\
\hline & & & $\begin{array}{c}\text { Clay } \\
\text { Minerals }\end{array}$ & & & & & & & & $\begin{array}{l}\text { Minera } \\
\quad=10\end{array}$ & $\begin{array}{l}\text { alogy } \\
00 \%\end{array}$ & \\
\hline & & & $\begin{array}{c}\text { Volcanic } \\
\text { Glass }\end{array}$ & Quartz & $\begin{array}{l}\text { Feld- } \\
\text { spar }\end{array}$ & Pyroxene & $\begin{array}{l}\text { Horn- } \\
\text { blende }\end{array}$ & $\begin{array}{l}\text { Carbon- } \\
\text { ate }\end{array}$ & $\begin{array}{l}\text { Clinop- } \\
\text { tilolite }\end{array}$ & $\begin{array}{l}\text { Anal- } \\
\text { cime }\end{array}$ & Smectite & Illite & \\
\hline Hole 451 & & & & & & & & & & & & & \\
\hline $1-2,9-11$ & 1 & 1.60 & 37.0 & 0.9 & 3.4 & - & $1-2$ & 55.8 & - & - & - & - & \\
\hline $2-3,98-100$ & 2 & - & - & - & - & - & $\bar{T}$ & $\overline{7 s 0}$ & - & - & - & $=$ & \\
\hline $3-3,102-104$ & 3 & 18.63 & 21.6 & - & 3,4 & - & Tr. & 75.0 & - & - & - & - & \\
\hline $4-3,72-74$ & 4 & - & - & - & - & - & - & - & - & - & $\overline{0}$ & - & \\
\hline $5-4,70-72$ & 5 & 38.71 & 68.8 & 0.9 & 10.0 & - & $1-2$ & 19.2 & - & - & 100 & - & \\
\hline $6-1,73-75$ & 6 & - & - & - & - & - & - & - & - & - & - & - & \\
\hline $8-6,33-35$ & 7 & 69.84 & 62.0 & 0.9 & 11.0 & - & $1-2$ & 25.6 & - & - & 100 & - & \\
\hline $14-2,57-59$ & 8 & - & - & - & - & - & - & - & - & - & - & - & \\
\hline $25-1,82-84$ & 9 & 224.33 & 64.1 & 1.0 & 9.4 & - & 2 & 13.5 & - & - & 100 & - & \\
\hline $27-1,53-55$ & 10 & - & - & - & - & - & - & - & - & - & - & - & \\
\hline $31-1,32-34$ & 11 & 280.83 & 42,3 & - & 8.0 & - & 1 & 48.7 & - & - & 100 & - & \\
\hline $32-1,120-122$ & 12 & $\overline{-}$ & - & - & - & - & - & $\overline{3}$, & - & - & $\overline{0}$ & - & \\
\hline $33-1,43-46$ & 13 & 299.95 & 78.9 & - & 11.4 & - & 2.5 & 3.2 & - & - & 100 & - & \\
\hline $34-1,133-135$ & 14 & - & - & - & - & - & - & - & - & - & $\overline{0}$ & - & \\
\hline $35-1,12-14$ & 15 & 318.63 & 84.0 & - & 8.8 & - & $1-2$ & 3.2 & - & - & 100 & - & \\
\hline $36-1,55-57$ & 16 & - & - & - & - & - & - & - & - & - & - & - & \\
\hline $37-1,7-10$ & 17 & 337.59 & 86.5 & $<1.0$ & 10.0 & $1-2$ & 2 & - & - & - & 100 & - & \\
\hline $38-2,138-140$ & 18 & - & - & - & - & - & - & - & - & - & - & - & \\
\hline $39-1,43-45$ & 19 & 356.94 & 86.0 & $<1.0$ & 9.0 & 2 & 2 & - & - & - & 100 & - & \\
\hline $39, \mathrm{CC}, 0-2$ & 20 & - & - & - & - & - & - & - & - & - & - & - & \\
\hline $40-1,87-90$ & 21 & 366.89 & 81.1 & $<1.0$ & 7.0 & 3 & $1-2$ & 6.4 & - & - & 100 & - & \\
\hline $41-1,82-84$ & 22 & $\overline{385} 64$ & $-\overline{848}$ & - & $\bar{s} 0$ & $\bar{T}$ & $\bar{T}$ & $\overline{10}$ & - & - & $\overline{0}$ & - & \\
\hline $\begin{array}{l}42-1,61-67 \\
42-1,10-13\end{array}$ & $\begin{array}{l}23 \\
24\end{array}$ & $\begin{array}{c}385.64 \\
-\end{array}$ & $\begin{array}{c}84.8 \\
-\end{array}$ & $\bar{z}$ & 5.0 & Tr. & Tr. & 10.2 & - & - & 100 & - & \\
\hline $\begin{array}{l}42-1,10-13 \\
45, C \text { C } 18-20\end{array}$ & $\begin{array}{l}24 \\
25\end{array}$ & $41 \overline{6.50}$ & $\overline{76.0}$ & $\overline{1.3}$ & $\overline{11.0}$ & $\overline{2}$ & $\overline{0}$ & $\bar{n}$ & - & - & $\overline{0}$ & - & \\
\hline $\begin{array}{l}45, \mathrm{CC}, 18-20 \\
52-1,69-71\end{array}$ & $\begin{array}{l}25 \\
26\end{array}$ & 416.50 & 76.0 & & & 2 & 2 & 7.7 & - & - & 100 & - & \\
\hline $\begin{array}{l}52-1,69-71 \\
53-1,72-74\end{array}$ & $\begin{array}{l}26 \\
27\end{array}$ & 474.23 & 915 & - & - & - & - & - & - & - & $\overline{0}$ & - & \\
\hline $53-1,72-74$ & $\begin{array}{l}27 \\
28\end{array}$ & 474.23 & $\begin{array}{l}91.5 \\
-\end{array}$ & - & 3.4 & Tr. & Tr. & 5.1 & - & - & 100 & - & \\
\hline $54-3,47-49$ & 28 & - & - & - & - & - & - & - & - & - & - & - & \\
\hline $55-2,22-24$ & 29 & 491.23 & 96.6 & - & 2.4 & Tr. & Tr. & - & - & - & 100 & - & \\
\hline $56-1,80-82$ & 30 & - & - & - & - & - & - & - & - & - & - & - & \\
\hline $58-2,55-57$ & 31 & 520.00 & 76.5 & - & 21.0 & - & 2.5 & Tr. & $\mathrm{Tr}$. & - & 100 & - & \\
\hline $60-1,48-50$ & 32 & - & - & - & - & - & - & - & - & - & - & - & \\
\hline $62-2,57-59$ & 33 & 558.08 & 77.0 & - & 7.0 & 1 & 3 & - & - & 6 & 100 & - & \\
\hline $63-4,99-103$ & 34 & 5090 & $\overline{70 .}$ & - & $\overline{0}$ & - & - & - & - & - & $\overline{0}$ & - & \\
\hline $63-5,37-42$ & 35 & 569.90 & 72.5 & - & 24.0 & - & 3.5 & - & - & - & 100 & - & \\
\hline $64-3,94-96$ & 36 & - & - & - & - & - & - & - & - & - & $\overline{-1}$ & - & \\
\hline $65-2,29-36$ & 37 & 586.33 & 76.4 & - & 20.6 & - & 3 & - & - & - & 100 & - & \\
\hline $67-1,28-30$ & 38 & - & - & - & - & - & - & - & - & - & - & - & \\
\hline $68-2,66-68$ & 39 & 615.16 & 74.8 & - & 15.6 & - & 2.5 & 7.1 & - & - & 100 & - & \\
\hline $70-1,32-34$ & 40 & - & - & - & - & - & - & - & - & - & - & - & \\
\hline $71-1,60-62$ & 41 & 642.11 & 81.5 & - & 15.0 & - & 3.5 & - & $\mathrm{Tr}$. & - & 100 & - & \\
\hline $72-3,52-54$ & 42 & - & - & - & - & - & - & - & - & - & - & - & \\
\hline $73-1,42-44$ & 43 & 660.93 & 72.5 & - & 13.0 & - & 1 & 13.5 & - & - & 100 & - & \\
\hline $73-1,116-118$ & 44 & 661.67 & - & - & - & - & - & - & - & - & - & - & \\
\hline $74-2,24-26$ & 45 & 671.75 & 84.8 & - & 7.2 & - & Tr. & - & - & 8 & 100 & - & \\
\hline $78-2,99-100$ & 46 & $\overline{-5} 87$ & - & - & - & - & - & - & - & - & - & - & \\
\hline $78-3,6-8$ & 47 & 705.57 & 83.2 & - & 14.8 & - & 2 & - & Tr. & - & 100 & - & \\
\hline $79, \mathrm{CC}, 31-33$ & 48 & - & - & - & - & - & - & - & - & - & $\overline{0}$ & - & \\
\hline $80-3,89-93$ & 49 & 721.41 & 82.0 & - & 16.0 & - & 2 & - & - & - & 100 & - & \\
\hline $81-1,115-117$ & 50 & - & - & - & - & - & - & - & - & - & - & - & \\
\hline $82-3,36-38$ & 51 & 739.87 & 86.5 & - & 12.0 & - & $1-2$ & - & $\operatorname{Tr}$. & - & 100 & - & \\
\hline $85-2,92-94$ & 52 & - & - & - & - & - & - & - & - & - & - & - & \\
\hline $86-3,89-92$ & 53 & 778.40 & 91.6 & - & 8.4 & - & - & - & $\mathrm{Tr}$. & - & 100 & - & \\
\hline $88-3,45-48$ & 54 & - & - & - & - & - & - & - & - & - & - & - & \\
\hline $89-1,129-132$ & 55 & 804.31 & 87.0 & - & 11.0 & - & 2 & - & Tr. & - & 100 & - & \\
\hline $91-2,12-17$ & 56 & - & - & - & - & - & - & - & - & - & - & - & \\
\hline $92-2,0-5$ & 57 & 833.03 & 84.5 & - & 13.0 & - & 2.5 & - & $\mathrm{Tr}$. & - & 100 & - & \\
\hline $94-1,1-4$ & 58 & - & - & - & - & - & - & - & - & - & - & - & \\
\hline $98-3,8-11$ & 59 & 891.60 & 82.0 & z & 15.0 & - & 3.0 & $\bar{z}$ & $\bar{z}$ & $\bar{z}$ & 100 & $\overline{-}$ & \\
\hline $\begin{array}{l}99-2,9-11 \\
100-1,148-149\end{array}$ & $\begin{array}{l}60 \\
61\end{array}$ & $\overline{908.98}$ & $\overline{69.2}$ & $\bar{z}$ & $\overline{8.4}$ & $\bar{z}$ & $\overline{2}$ & 19.2 & 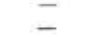 & $=$ & 80.2 & 19.8 & \\
\hline
\end{tabular}

${ }^{a}$ Not separated due to small amounts. 
Table 2. (Continued).

\begin{tabular}{|c|c|c|c|c|c|c|c|c|c|c|c|c|}
\hline \multirow[b]{3}{*}{$\begin{array}{l}\text { Core-Section } \\
\text { (interval in } \mathrm{cm} \text { ) }\end{array}$} & \multirow[b]{3}{*}{ Sample } & \multirow[b]{3}{*}{$\begin{array}{l}\text { Depth } \\
\text { (m) }\end{array}$} & \multicolumn{8}{|c|}{ Bulk Mineralogy $=100 \%$} & \multirow{2}{*}{\multicolumn{2}{|c|}{$\begin{array}{c}\text { Clay } \\
\text { Mineralogy } \\
=100 \%\end{array}$}} \\
\hline & & & \multirow{2}{*}{$\begin{array}{c}\text { Clay } \\
\text { Minerals } \\
+ \text { Volcanic } \\
\text { Glass }\end{array}$} & \multirow[b]{2}{*}{ Quartz } & \multirow[b]{2}{*}{$\begin{array}{l}\text { Feld- } \\
\text { spar }\end{array}$} & \multirow[b]{2}{*}{ Pyroxene } & \multirow[b]{2}{*}{$\begin{array}{l}\text { Horn- } \\
\text { blende }\end{array}$} & \multirow[b]{2}{*}{$\begin{array}{l}\text { Carbon- } \\
\text { ates }\end{array}$} & \multirow[b]{2}{*}{$\begin{array}{l}\text { Clinop- } \\
\text { tilolite }\end{array}$} & \multirow[b]{2}{*}{ Analcime } & & \\
\hline & & & & & & & & & & & Smectite & Illite \\
\hline Hole 450 (Cont.) & & & & & & & & & & & & \\
\hline $14-6,115-117$ & 13 & 130.16 & 87.5 & Tr. & 6.0 & 1 & 1 & 4.5 & - & 100 & - & - \\
\hline $15-2,128-130$ & 14 & - & - & - & - & - & - & - & - & - & - & - \\
\hline $16-1,46-47$ & 15 & 140.96 & 85.4 & -1 & 6.6 & $1-2$ & 5 & 4.5 & - & 100 & - & - \\
\hline $17-3,24-26$ & 16 & - & - & - & - & - & - & - & - & - & - & - \\
\hline $18-1,26-28$ & 17 & 159.77 & 84.7 & $\sim 1$ & 5.4 & $1-2$ & -1 & 6.4 & - & 100 & - & - \\
\hline $19-6,14-17$ & 18 & - & - & - & - & - & - & - & - & - & - & - \\
\hline $20-2,13-15$ & 19 & 180.14 & 85.5 & -1 & 5.4 & $1-2$ & $1-2$ & 5.1 & - & 100 & - & - \\
\hline $20-3,14-16$ & 20 & - & - & - & - & - & - & - & - & - & - & - \\
\hline $21-4,51-53$ & 21 & 193.02 & 88.6 & - & 3.0 & 1 & 1 & 6.4 & - & 100 & - & - \\
\hline $22-6,6-8$ & 22 & - & - & - & - & - & - & - & - & - & - & - \\
\hline $24-3,10-12$ & 23 & 219.61 & 81.1 & 1.3 & 10.0 & $1-2$ & 1 & 5.1 & - & 100 & - & - \\
\hline $26-2,41-43$ & 24 & - & - & - & - & - & - & - & - & - & - & - \\
\hline $29-3,49-52$ & 25 & 267.51 & 82.7 & 1.3 & 6.0 & $1-2$ & $1-2$ & 7.0 & - & 100 & - & - \\
\hline $30-3,33-35$ & 26 & - & - & - & - & - & - & - & - & - & - & - \\
\hline $32-3,84-87$ & 27 & 296.36 & 84.0 & -1 & 5.6 & 1 & 1 & 10.2 & - & 100 & - & - \\
\hline $33-1,123-124$ & 28 & - & - & - & - & - & - & - & - & - & - & - \\
\hline $35-3,83-85$ & 29 & 324.84 & 95.2 & - & -1 & - & - & 3.2 & - & 100 & - & - \\
\hline $36-1,7-10$ & 30 & - & - & - & - & - & - & - & - & - & - & - \\
\hline $36-1,148-150$ & 31 & 331.99 & 81.7 & 2.1 & 3.4 & - & - & 12.8 & - & 100 & - & - \\
\hline $36-2,118-122$ & 32 & - & - & - & - & - & - & - & - & - & - & - \\
\hline
\end{tabular}

\section{Hole 451}

1-2, 9-11

$2-3,98-100$

3-3, 102-104

$4-3,72-74$

$5-4,70-72$

6-1, 73-75

$8-6,33-35$

14-2, 57-59

$25-1,82-84$

27-1, 53-55

$31-1,32-34$
$32-1,120-122$

$32-1,120-122$
$33-1,43-46$

$34-1,133-135$

$35-1,12-14$

$36-1,55-57$

$37-1,7-10$

$38-2,138-140$

39-1, 43-45

$39, \mathrm{CC}, 0-2$

40-1, 87-90

$41-1,82-84$

$42-1,61-67$

42-1, 10-13

45, CC, $18-20$

$52-1,69-71$

$53-1,72-74$

$54-3,47-49$

$55-2,22-24$

$56-1,80-82$

$58-2,55-57$

$60-1,48-50$

62-2, 57-59

$63-4,99-103$

$63-5,37-42$

64-3, 94-96

$65-2,29-36$

$67-1,28-30$

68-2, 66-68

$70-1,32-34$

$71-1,60-62$

$72-3,52-54$

$73-1,42-44$

$73-1,116-118$

$74-2,24-26$

$78-2,99-100$

$78-3,6-8$

$79, \mathrm{CC}, 31-33$

80-3, 89-93

$81-1,115-117$

$82-3,36-38$

$85-2,92-94$

$86-3,89-92$

$88-3,45-48$

$89-1,129-132$

$91-2,12-17$

$92-2,0-5$

$94.1,1-4$

$98-3,8-11$

$99-2,9-11$

$100-1,148-149$

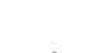

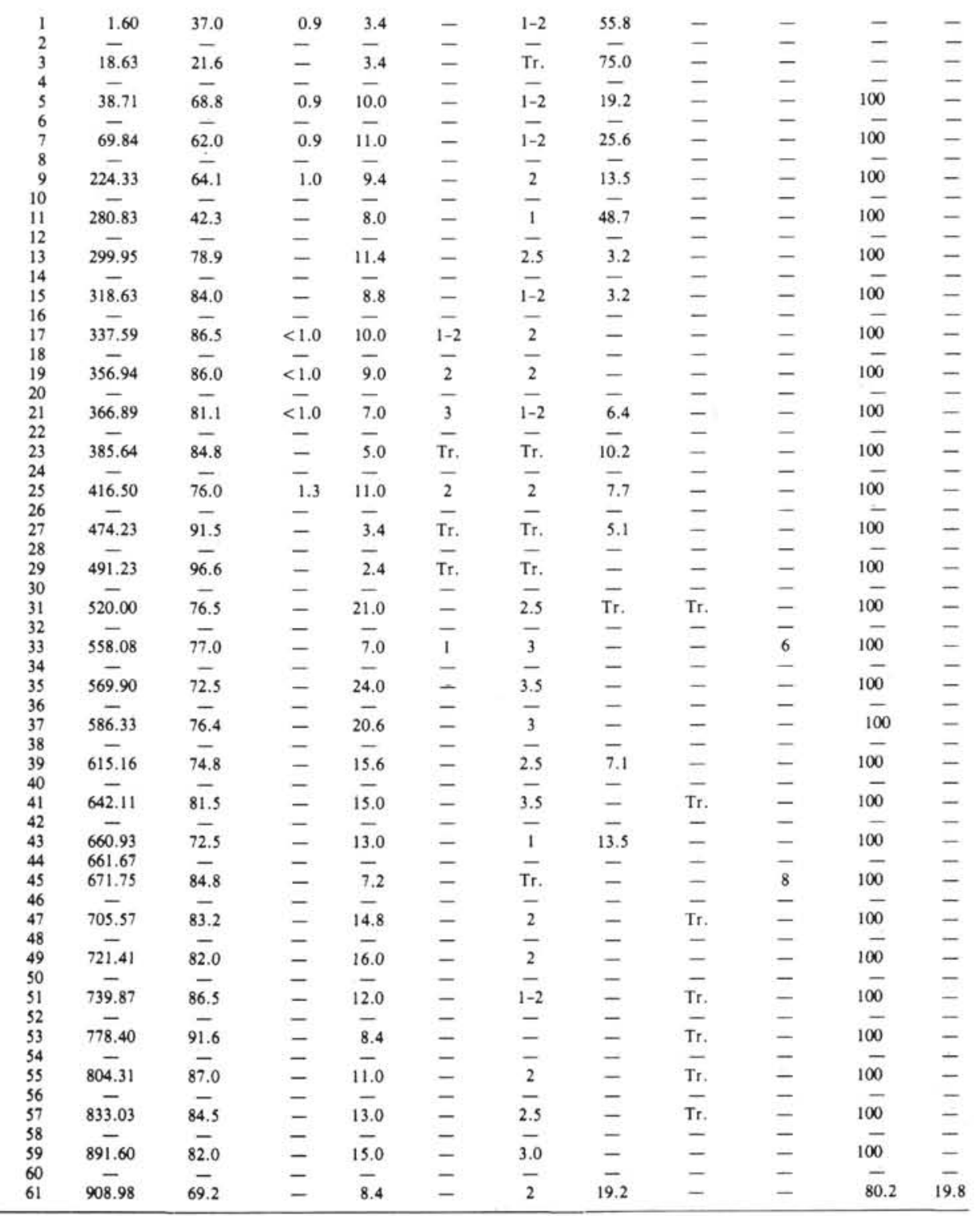

a Not separated due to small amounts. 
Table 3. Summary of X-ray mineralogy samples, sample depths; and X-ray diffraction results of Sites 453-461, Leg 60.

\begin{tabular}{|c|c|c|c|c|c|c|c|c|c|c|c|c|c|c|}
\hline \multirow[b]{2}{*}{$\begin{array}{l}\text { Core-Section } \\
\text { (interval in cm) }\end{array}$} & \multirow[b]{2}{*}{ Sample } & \multirow[b]{2}{*}{$\begin{array}{c}\text { Depth } \\
\text { (m) }\end{array}$} & \multicolumn{8}{|c|}{ Bulk Mineralogy $=100 \%$} & \multicolumn{4}{|c|}{ Clay Mineralogy $=100 \%$} \\
\hline & & & $+\begin{array}{l}\text { Volcanic } \\
\text { Glass }\end{array}$ & Quartz & $\begin{array}{l}\text { Feld- } \\
\text { spar }\end{array}$ & Pyroxene & $\begin{array}{l}\text { Horn- } \\
\text { blende }\end{array}$ & $\begin{array}{l}\text { Carbon- } \\
\text { ate }\end{array}$ & Phillipsite & Other & Smectite & Illite & Chiorite & Kaolinite \\
\hline \multicolumn{15}{|l|}{ Hole 453} \\
\hline $3-2,40-42$ & 1 & 20.41 & 87.6 & 1.8 & 7.6 & $1-2$ & $1-2$ & - & - & - & 55.6 & 37.1 & - & 7.3 \\
\hline $5-4,138-140$ & 2 & 43.39 & 89.9 & 1.0 & 5.6 & $1-2$ & 2.5 & - & - & - & 76.9 & - & - & 23.1 \\
\hline $6-1,130-132$ & 3 & 48.31 & 89.0 & 1.6 & 7.4 & 1 & 1 & - & - & - & 100.0 & - & - & - \\
\hline $9-1,23-25$ & 4 & 75.74 & 85.9 & 1.3 & 8.8 & 2 & 1 & - & - & - & 95.4 & - & - & 4.6 \\
\hline $10-2,89-92$ & 5 & 87.40 & 89.5 & 1.0 & 3.6 & $<1$ & $<1$ & 3.9 & - & - & 97.8 & - & - & 2.2 \\
\hline $11-4,70-74$ & 6 & 99.72 & 87.5 & 2.5 & 7.0 & $1-2$ & $1-2$ & - & - & - & 97.5 & - & - & 2.5 \\
\hline $15-1,24-26$ & 7 & 132.75 & 87.1 & 1.9 & 6.0 & $<1$ & - & - & 4.0 & - & 94.4 & - & 1.6 & 4.0 \\
\hline $18-1,30-32$ & 8 & 161.31 & 88.7 & 1.7 & 4.6 & 2 & - & - & 3.0 & - & 95.0 & - & - & 5.0 \\
\hline $18-2,24-26$ & 9 & 162.75 & 78.0 & 1.0 & $5-6$ & - & - & 8.4 & 7.0 & - & 97.2 & - & 1.0 & 1.8 \\
\hline $19-1,23-25$ & 10 & 170.74 & 93.8 & 1.3 & 3.4 & $1-2$ & - & - & - & - & 56.6 & 18.8 & - & 24.5 \\
\hline $22-2,83-85$ & 11 & 201.34 & 94.9 & 1.3 & 2.8 & 1 & - & - & - & - & 89.5 & - & - & 10.5 \\
\hline $23-1,86-88$ & 12 & 209.37 & 97.6 & - & 2.4 & - & - & - & - & - & 100.0 & - & - & - \\
\hline $23-1,96-98$ & 13 & 209.47 & 99.0 & - & 1.0 & 1 & Tr. & - & - & - & 100.0 & - & - & - \\
\hline $27-2,42-44$ & 14 & 248.43 & 91.7 & 1.3 & 4.0 & $1-2$ & $1-2$ & - & - & - & 95.5 & - & - & 4.9 \\
\hline $28-2,39-41$ & 15 & 257.90 & 89.2 & 1.3 & 4.0 & Tr. & $<1$ & 4.5 & - & - & 97.7 & - & - & 2.3 \\
\hline $29-2,53-55$ & 16 & 267.54 & 89.7 & 1.3 & 7.0 & 1 & 1 & - & - & - & 93.0 & - & - & 7.0 \\
\hline $33-4,11-12$ & 17 & 308.11 & 90.2 & 3.0 & 4.8 & $<1$ & 1 & - & - & - & 98.2 & - & 0.5 & 1.3 \\
\hline $35-3,41-43$ & 18 & 325.92 & 91.2 & 1.0 & 6.8 & Tr. & Tr. & - & 2.0 & - & 99.1 & - & 0.6 & 0.3 \\
\hline $39-1,136-140$ & 19 & 361.88 & 85.5 & 1.0 & 6.0 & - & 2.5 & - & - & $5^{\mathrm{a}}$ & 98.6 & - & 0.7 & 0.7 \\
\hline $42-2,13-15$ & 20 & 390.64 & 86.5 & 1.0 & 10.0 & 1 & $1-2$ & - & - & - & 100.0 & - & - & - \\
\hline $46-1,106-108$ & 21 & 428.07 & 93.0 & 1.0 & 6.0 & - & 1 & - & - & - & 100.0 & - & - & - \\
\hline $48-1,27-29$ & 22 & 446.28 & 92.5 & 2.0 & 4.0 & $1-2$ & - & - & - & - & 100.0 & - & - & - \\
\hline \multicolumn{15}{|l|}{ Hole 454} \\
\hline $4-5,22-24$ & 1 & 25.73 & 84.8 & 1.6 & 8.4 & - & 2 & 3.2 & - & - & 82.7 & - & & \\
\hline S,CC, $13-15$ & 2 & 38.44 & 90.6 & 1.0 & 5.4 & - & 1 & - & - & - & 75.0 & - & & 25.0 \\
\hline \multicolumn{15}{|l|}{ Hole 454A } \\
\hline $2-2,20-24$ & 3 & 40.22 & 97.0 & - & 1.0 & - & 2 & - & - & - & 65.2 & - & & 34.8 \\
\hline \multicolumn{15}{|l|}{ Hole 456} \\
\hline $\begin{array}{l}3-2,20-24 \\
7-2\end{array}$ & $\frac{1}{2}$ & 11.72 & 82.1 & 0.9 & 8.4 & 2 & $1-2$ & 5.1 & - & $\overline{-}$ & 85.6 & $z$ & & 14.5 \\
\hline $7-2,29-31$ & 2 & 49.80 & 79.4 & 1.7 & 8.0 & 2 & 2.5 & 6.4 & - & - & 68.0 & - & & 32.0 \\
\hline $15, \mathrm{CC}, 0-4$ & 3 & 133.34 & 59.8 & 17.5 & 6.4 & - & 2.8 & 13.5 & - & - & 26.4 & - & - & 73.6 \\
\hline \multicolumn{15}{|l|}{ Hole 456A } \\
\hline $1-7,22-24$ & 4 & 9.23 & 89.0 & 1.0 & 7.0 & 2 & 1 & - & - & - & 50.0 & - & & 50.0 \\
\hline $3-3,20-22$ & 5 & 41.21 & 84.6 & 1.0 & 2.4 & - & 1 & - & - & $\overline{c h}$ & 90.0 & - & & 10.0 \\
\hline $7-1,54-56$ & 6 & 76.55 & 71.6 & - & 3.4 & $\operatorname{Tr}$. & Tr. & - & - & $25^{\mathrm{b}}$ & 100.0 & - & - & - \\
\hline Hole 458 & & & & & & & & & & & & & & \\
\hline $2-6,69-71$ & 1 & 17.70 & 88.5 & - & 10.0 & - & Tr. & - & - & - & 100.0 & - & - & $1-2$ \\
\hline $11-3,13-14$ & 2 & 98.14 & 69.0 & 0.9 & 10.0 & - & $1-2$ & 16.0 & - & - & - & - & - & $2-3$ \\
\hline Hole 459B & & & & & & & & & & & & & & \\
\hline $1-1,139-140$ & 1 & 1.40 & 72.4 & 2.6 & 20.0 & 1 & 3 & - & - & - & 83.3 & - & 5.8 & 10.8 \\
\hline $1-2,70-71$ & 2 & 2.20 & 76.0 & 1.3 & 14.0 & 2 & 2 & 3.2 & - & - & 49.0 & 24.5 & 8.3 & 18.1 \\
\hline $2-3,60-63$ & 3 & 11.12 & 80.1 & 2.6 & 7.6 & 1 & 1 & 7.7 & - & - & 71.4 & 16.3 & - & 12.3 \\
\hline $11-1,80-82$ & 4 & 93.81 & 85.4 & 1.3 & 7.0 & $1-2$ & 1 & 3.8 & - & - & 94.2 & - & 0.7 & 5.1 \\
\hline $13-1,32-34$ & 5 & 112.83 & 79.6 & 1.7 & 11.0 & 2 & 2.5 & 3.2 & - & - & 100.0 & - & - & - \\
\hline $21-1,70-72$ & 6 & 196.21 & 93.4 & 2.6 & 4.0 & Tr. & Tr. & - & - & - & 100.0 & - & - & - \\
\hline $24-2,84-86$ & 7 & 218.85 & 80.5 & 0.9 & 11.0 & $1-2$ & 1 & 5.1 & - & - & 100.0 & - & - & - \\
\hline $25-1,26-28$ & 8 & 226.27 & 87.2 & 1.0 & 7.0 & 1 & Tr. & 3.8 & - & - & 100.0 & - & - & - \\
\hline $26-1,61-63$ & 9 & 236.12 & 78.5 & 2.0 & 13.0 & 2.5 & 1.5 & - & - & - & 100.0 & - & - & - \\
\hline $27-4,53-55$ & 10 & 250.04 & 89.2 & 1.0 & 4.6 & 1 & 1 & 3.2 & - & - & 100.0 & - & - & - \\
\hline $44-2,4-7$ & 11 & 408.06 & 84.0 & 2.0 & 11.0 & 1 & 2 & - & - & - & 100.0 & - & - & - \\
\hline $51-1,65-67$ & 12 & 473.66 & 89.2 & 2.2 & 7.6 & 1 & Tr. & - & - & - & 100.0 & - & - & - \\
\hline $58-2,40-42$ & 13 & 541.41 & 80.5 & 1.0 & 11.0 & $4-5$ & 3 & - & - & - & 100.0 & - & - & - \\
\hline Hole 460 & & & & & & & & & & & & & & \\
\hline $3-2,50-52$ & 1 & 19.51 & 87.1 & 1.3 & 7.8 & $1-2$ & $1-2$ & - & - & - & 49.2 & 18.9 & 26.4 & 5.5 \\
\hline Hole $460 \mathrm{~A}$ & & & & & & & & & & & & & & \\
\hline $4-7,18-20$ & 2 & 36.19 & 85.1 & 2.3 & 5.4 & 2 & 2 & 3.2 & - & - & 45.5 & 9.0 & & $45.5^{\mathrm{c}}$ \\
\hline $5-3,80-82$ & 3 & 40.31 & 80.3 & 5.7 & 7.0 & Tr. & 1 & - & 6 & - & 34.5 & 26.0 & 26.3 & 13.1 \\
\hline $8, C C, 21-23$ & 4 & 74.22 & 84.0 & - & 8 & Tr. & 2 & - & 8 & - & 33.3 & 61.8 & & 4.3 \\
\hline $8, \mathrm{CC}, 33-35$ & 5 & 74.44 & 83.5 & - & 14.0 & $1-2$ & Tr. & - & - & - & 22.4 & 77.6 & - & - \\
\hline Hole 461 & & & & & & & & & & & & & & \\
\hline $2-3,40-42$ & 1 & 4.91 & 80.8 & 2.6 & 8.0 & $1-2$ & $2-3$ & 2.6 & - & - & 40.7 & 35.8 & 17.4 & 6.0 \\
\hline
\end{tabular}

a Clinoptilotite.

b Analcime.

c Measured together as further separation was not possible. 


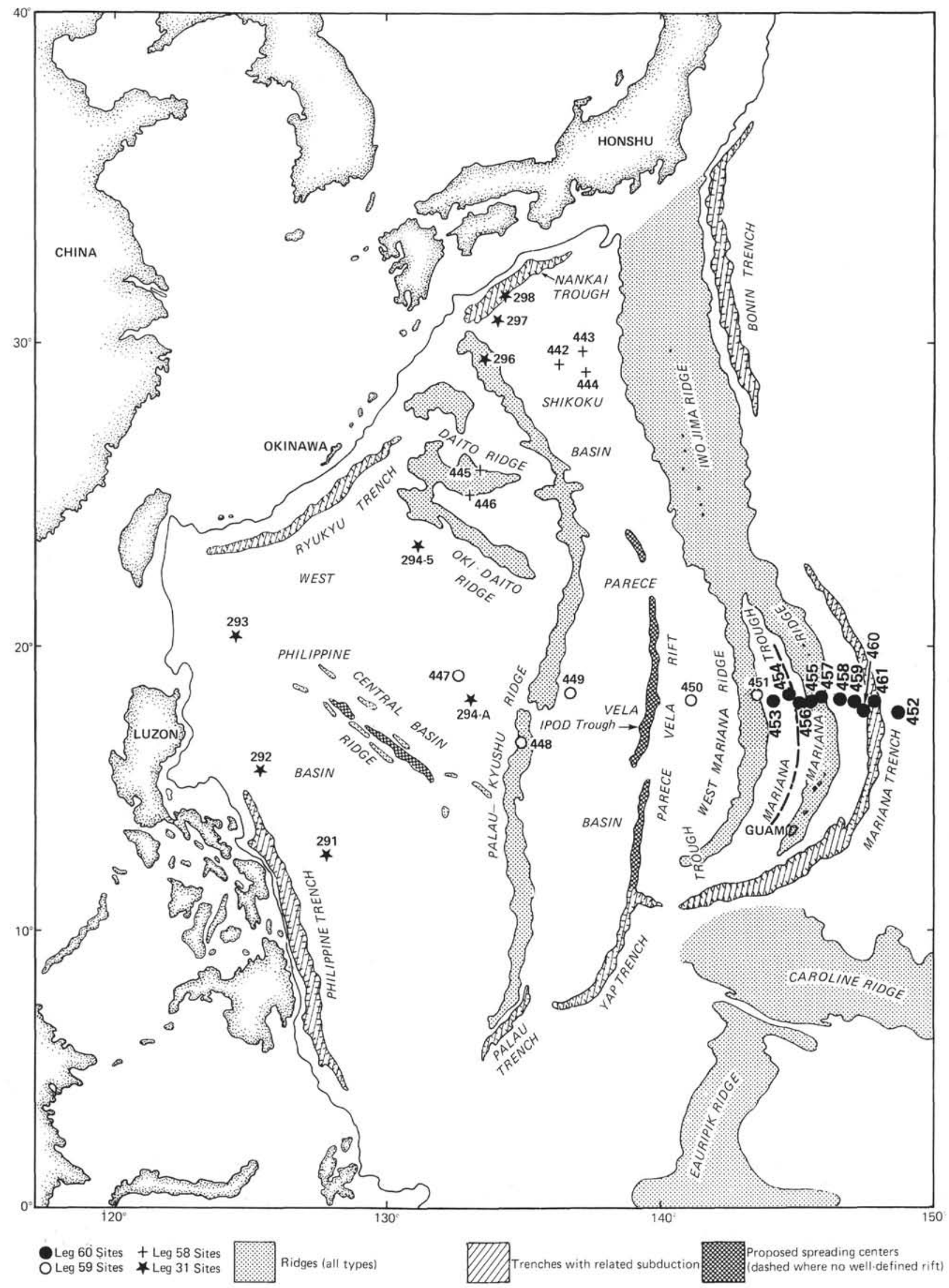

Figure 1. Locations of sites drilled on Legs 58 (Sites 442-446), 59 (Sites 447-451), and 60 (Sites 452-461), DSDP. 
Hole 442 / 442 A
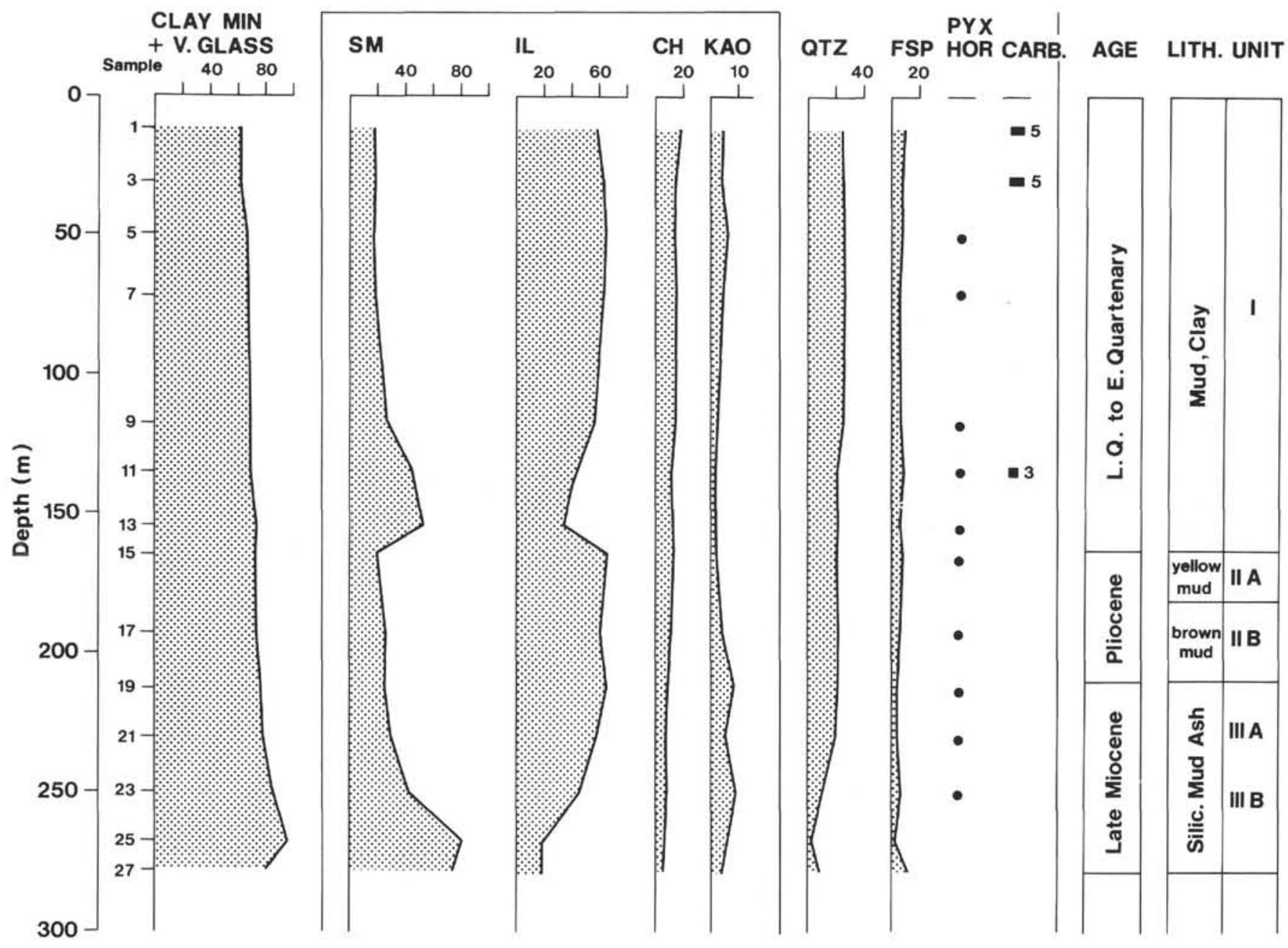

Figure 2. Hole 442: Semiquantitative determination of the mineral composition plotted versus depth below sea floor and lithology. Abbreviations used in this and the following figures are: $\mathrm{SM}=$ smectite, $\mathrm{IL}=$ illite, $\mathrm{CH}=$ chlorite, $\mathrm{KAO}=$ kaolinite, $\mathrm{QTZ}=$ quartz, FSP $=$ feldspar, $\mathrm{PYX}$ $=$ pyroxene, $\mathrm{HOR}=$ hornblende, $\mathrm{CARB}=$ carbonates, $\mathrm{CT}=$ opal-CT, $\mathrm{CPT}=$ clinoptilolite, $\mathrm{A}=$ analcime, $\mathrm{CB}=\mathrm{cristobalite}, \mathrm{PH}=$ phillipsite.

be 84 percent; a gradual decrease to 57 percent (Sample $29-2,79-81 \mathrm{~cm})$ is accompanied by rising clay mineral percentages (from $\sim 23$ to $\sim 36 \%$ ).

Unit IIB-middle Miocene to early Miocene very pale brown to brown clayey nannofossil chalk and (minor) calcareous siliceous mudstone-is characterized by carbonate contents varying between 71 and 57 percent and clay minerals between 22 and 36 percent. Unit IIC of early Miocene to middle Oligocene age is a pinkish white, gray, or pale brown, clayey nannofossil chalk. $\mathrm{CaCO}_{3}$ can reach 90 percent. Quartz and feldspar could not be detected in some samples. The main constituent of the $<2-\mu \mathrm{m}$ fraction is smectite; some samples contain no additional clay minerals (see Fig. 5).

Unit IID-middle Oligocene to early Oligocene pinkish white to pinkish gray, clayey nannofossil chalk-has a higher clay content than Unit IIC (up to $32 \%$ compared to $\sim 15 \%$ in Unit IIC). $\mathrm{CaCO}_{3}$ decreases from $\sim 88$ to $\sim 63$ percent. Late Eocene, gray to olive-gray, siliceous nannofossil chalk of Unit III contains still lesser amounts of $\mathrm{CaCO}_{3}$, varying between 35 and 72 percent. Opal A was found in Sample 61-2, 109-111 cm. All $<2-\mu \mathrm{m}$ samples of Unit III contain smectite as the only clay mineral.

Unit IV of middle Eocene age is a brown to reddish brown or greenish gray nannofossil or clayey radiolarite. $\mathrm{CaCO}_{3}$ is still an important constituent, although its content decreases to 26 percent. This (low $\mathrm{CaCO}_{3}$ ) sample also contains clinoptilolite in addition to the "normal" suite of clay minerals. In Sample 445-68-2, $41-43 \mathrm{~cm}$ (Unit IV) opal CT was found.

Unit VA (middle Eocene) is a greenish gray, partly sandy mudstone. Lithologies include sandstone and conglomerates, but none of these has as yet been X-rayed. Calcite contents of the mudstone samples vary between $\sim 14$ and $\sim 34$ percent, and clay minerals reach $>60$ percent. The $<2-\mu \mathrm{m}$ fraction is dominated by smectite, whereas illite was more abundant than smectite in the 
Hole 443

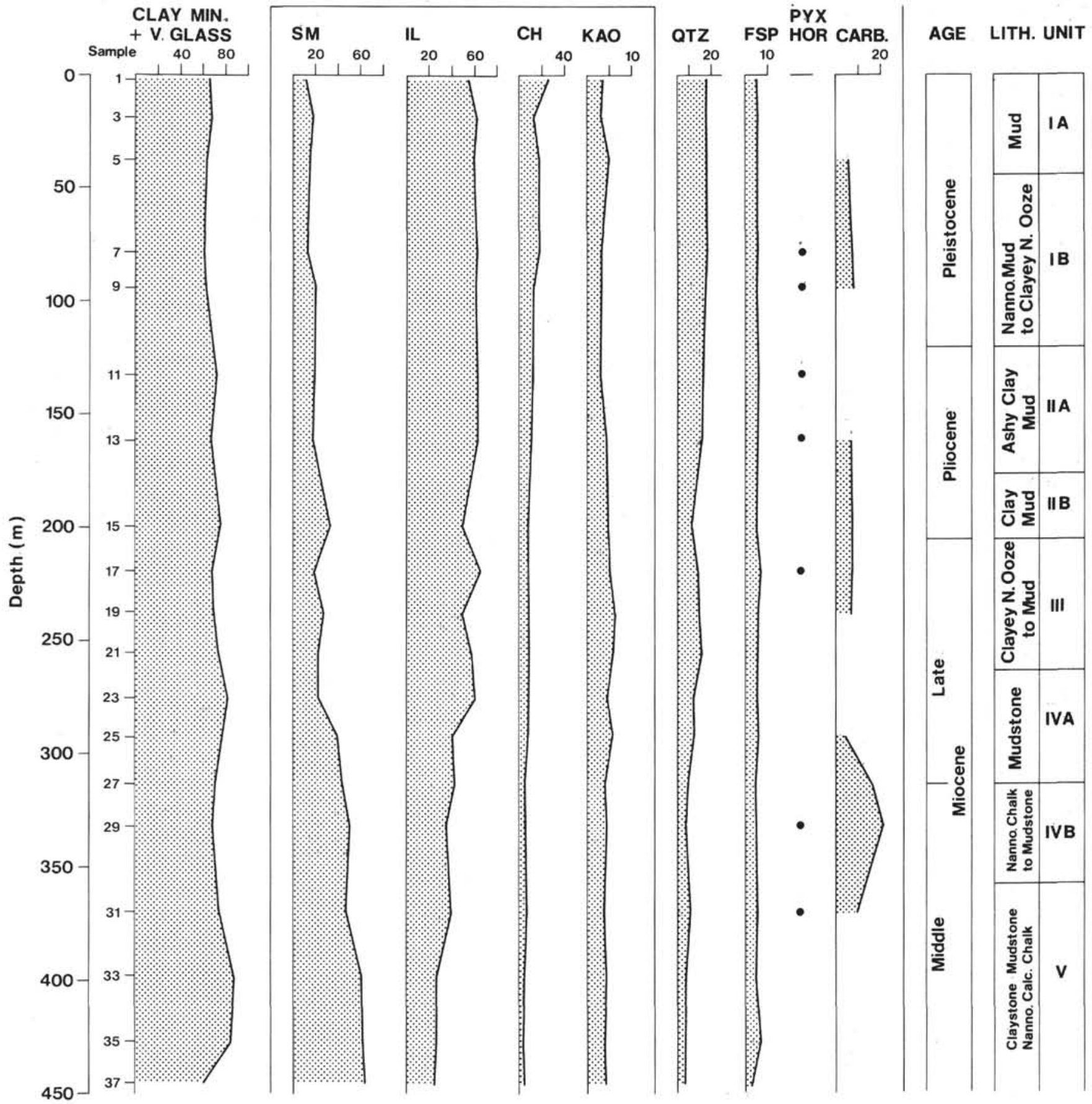

Figure 3. Hole 443: Semiquantitative determination of the mineral composition plotted versus depth below sea floor and lithology. Abbreviations as in Figure 2.

tite in the upper parts of Hole 445. Clinoptilolite was found in three of the analyzed samples; it is confined to the upper part of Unit VA.

Samples of Unit VB-a mudstone or silty mudstone (at base conglomeratic)-are characterized by decreasing $\mathrm{CaCO}_{3}$ contents $(<10 \%)$, whereby the clay mineral content is noticeable (ca. $80 \%$ ). No chlorite and illite could be detected whereas kaolinite was, and it varied between $\sim 10$ and $\sim 16$ percent. This is in contrast to Unit VA, where the usual clay minerals have been found, except for the lowest part that contains smectite only. One sample contained analcime.

Unit VI-middle Eocene-is a sequence dominated by a conglomerate. The color is dark greenish gray. 


\section{Hole 444/444 A}
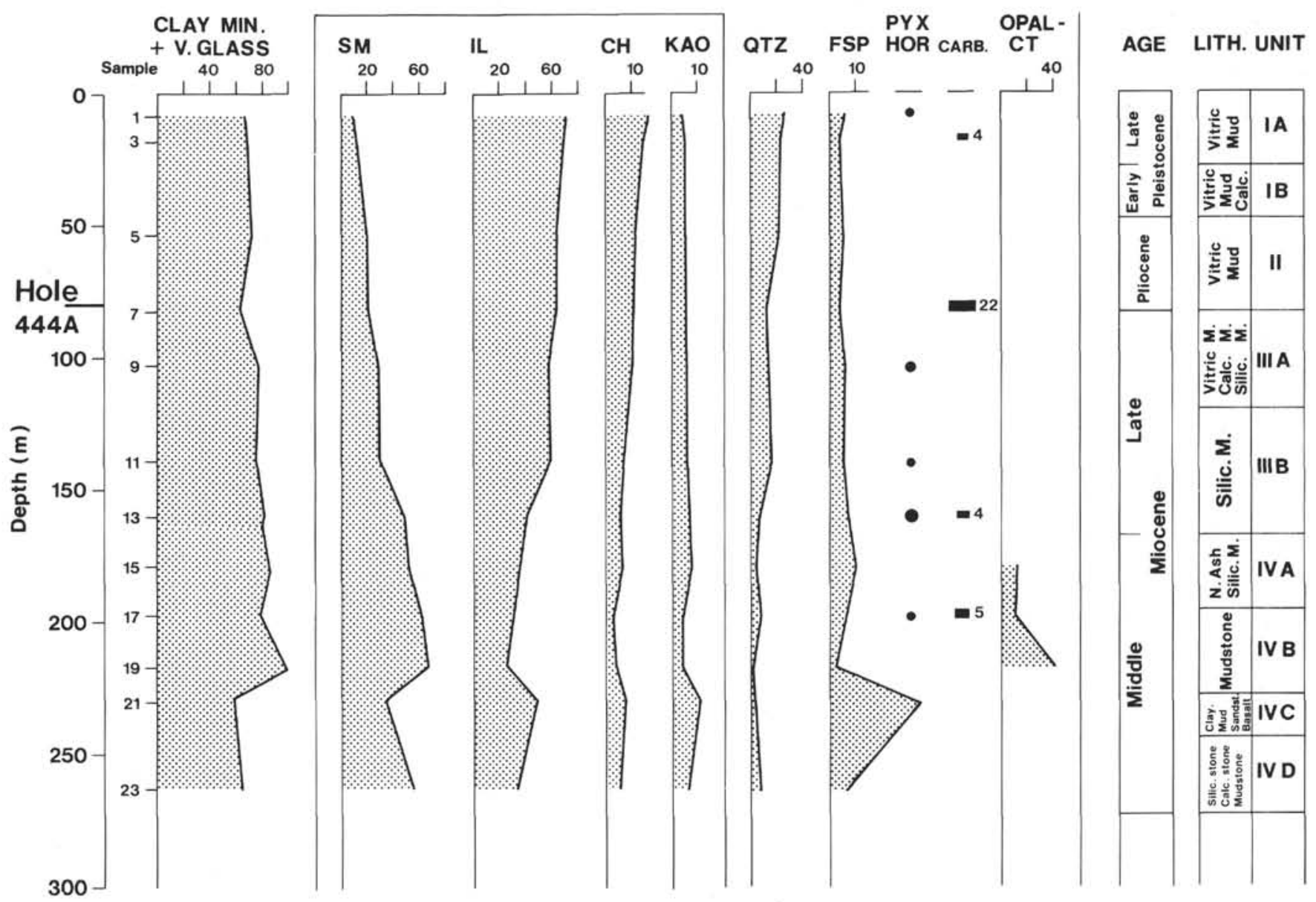

Figure 4. Holes 444 and 444A: Semiquantitative determination of the mineral composition plotted versus depth below sea floor and lithology. Abbreviations as in Figure 2 .

upper parts of Hole 445. Clinoptilolite was found in three of the analyzed samples; it is confined to the upper part of Unit VA.

\section{Site 446 (see Fig. 6)}

This site is situated in the Daito Basin, south of the Daito Ridge. 628.5 meters of sediment has been penetrated, the lower part of which by 16 basalt sills. Unit I, a Pliocene brown mud of terrigenous origin, consists of quartz $(\sim 26 \%)$, feldspar $(5 \%)$, and clay minerals inclusive amorphous constituents $(67 \%)$, as well as origin, consists of quartz $(\sim 26 \%)$, feldspar $(5 \%)$, and clay minerals inclusive amorphous constituents $(67 \%)$, as well as small amounts of hornblende and pyroxene. The clay mineral suite is dominated by illite $(64 \%)$. The upper section of Unit II is composed mainly of Miocene to Eocene pelagic brown to dark brown mud. Quartz and feldspar are abundant in the $>2-\mu \mathrm{m}$ fraction; clay minerals ( + amorphous material) are as high as 92 percent in Sample 446-9-2, 97-99 cm, which was described as "ashy clay."
This section is followed by a dark grayish brown pelagic clay section with almost constant amounts of detritals throughout its length $(\sim 6 \%)$ and clay minerals $(\sim 90 \%)$, of which smectite is the most prevalent. All increasing amounts of smectite again correspond to decreasing illite contents, of which only traces have been found in Sample 16-12, 38-40 cm. Sample 446-19-1, $147-149 \mathrm{~cm}$ contains 11 percent clinoptilolite, which has not been detected in samples from the upper part.

Unit IIA of middle Eocene age is a sequence of dark greenish gray or greenish gray, turbiditic mudstone as well as clay-, silt-, and sandstones. Only mudstone samples-typically comprising $80-90$ percent clayey material-have been X-rayed, the first of which (Sample $21-1,88-90 \mathrm{~cm}$ ) contains in addition to the "usual" suite of clay minerals, 2 percent clinoptilolite. The second sample (Sample 24-3, 126-129 $\mathrm{cm}$ ) has 3 percent $\mathrm{CaCO}_{3}$, but the clay minerals are once again dominant $(\sim 80 \%)$.

Sample $26-2,109-111 \mathrm{~cm}$, is an analcime-bearing mudstone ( $7 \%$ analcime). Also other samples of Unit 


\section{Hole 445}

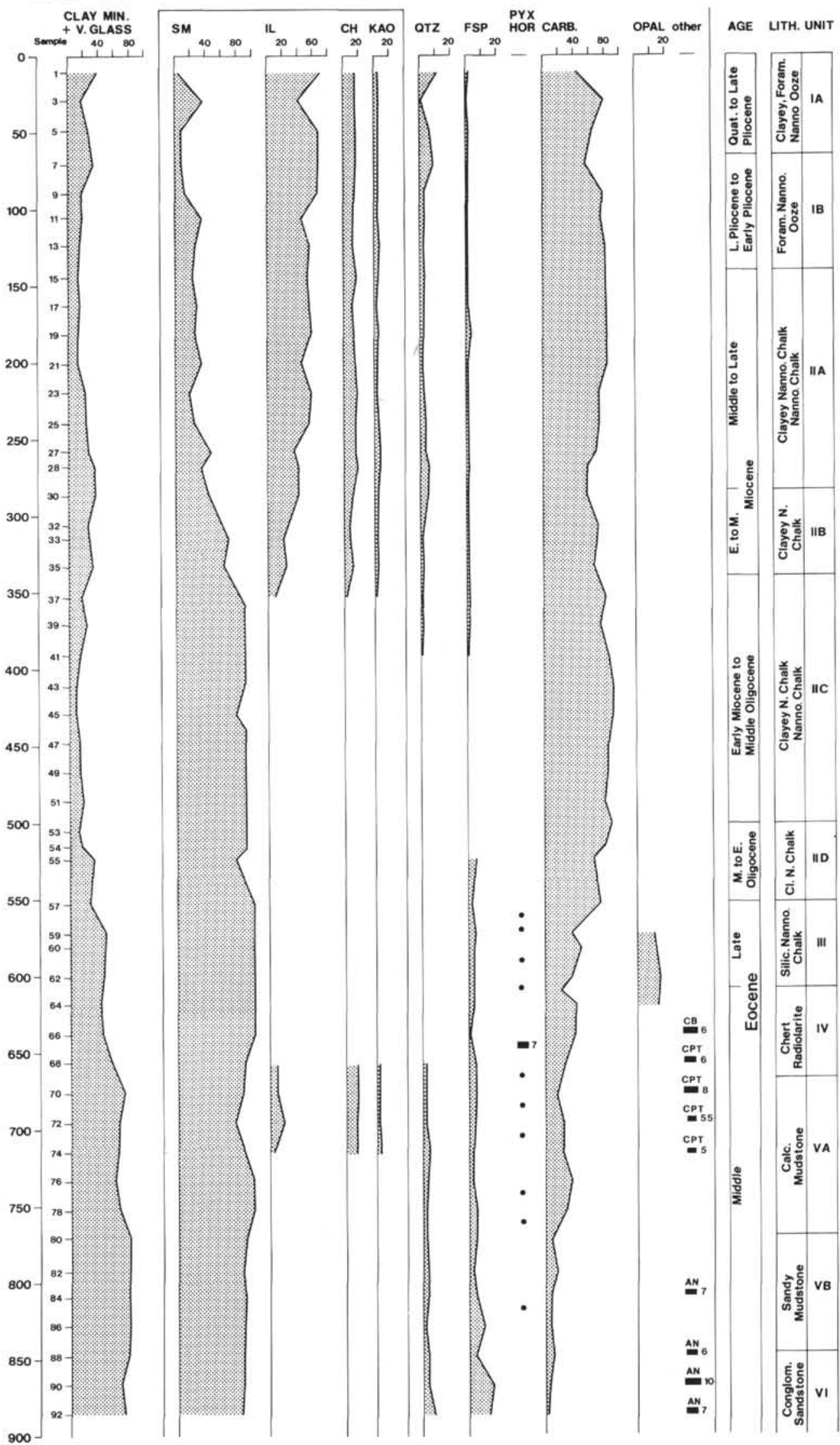

Figure 5. Hole 445: Semiquantitative determination of the mineral composition plotted versus depth below sea floor and lithology. Abbreviations as in Figure 2. 


\section{Holes 446/446 A}

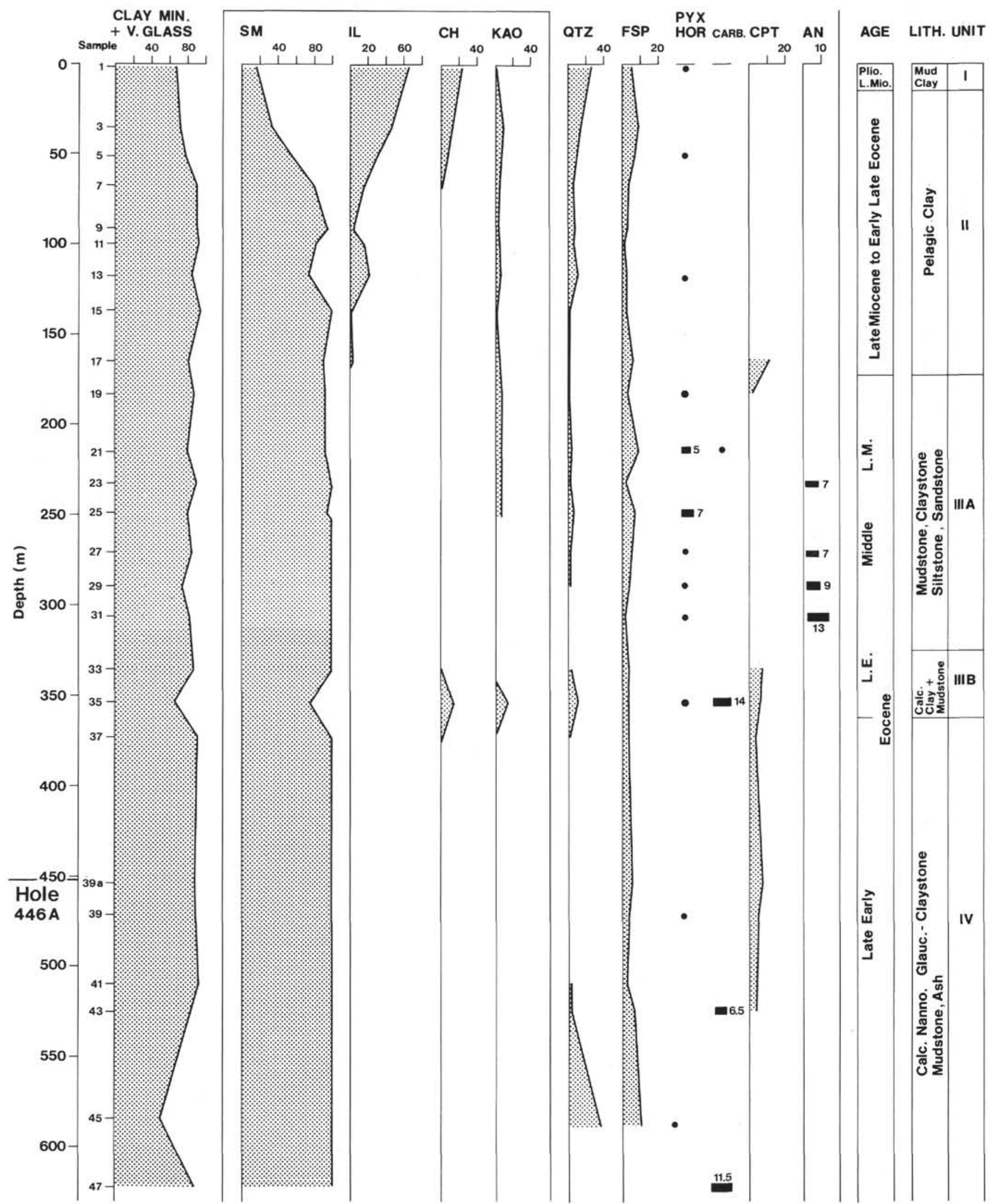

Figure 6. Hole 446 and 446A: Semiquantitative determination of the mineral composition plotted versus depth below sea floor and lithology. Abbreviations as in Figure 2. 
IIIA contain analcime; however, Units IIIB and IV are devoid of this mineral. Analcime, in addition to clinoptilolite, has been detected in Sample 32-2, 98-100 cm. In this sample a tiny fissure was found with native copper, Fe hydroxides, and quartz crystals.

Early Eocene brown to dark brown or grayish brown mudstone (Unit IIIB) is characterized by clinoptilolite $(\sim 7 \%)$ and smectite; however, no additional clay minerals have been found (Sample 37-1, 125-126 cm). Sample $39-1,26-28 \mathrm{~cm}$ has 14 percent $\mathrm{CaCO}_{3}$ and chlorite, kaolinite, and quartz; in the lowest part of Unit IIIA this detrital suite has not been detected.

Unit IV is a sequence of mainly dark greenish gray, calcareous claystones, mudstone, and ash of lower Eocene age. Chlorite, kaolinite, and illite have not been found in the $<2-\mu \mathrm{m}$ fractions of the samples analyzed; only smectite was yielded. Samples vary in quartz content between $<1$ percent and $\sim 36$ percent, and feldspar is also quite variable ( $2-10 \%)$. The high quartz/feldspar sample (Sample 446A-24-2, 23-24 cm) probably reflects a sedimentation that was influenced by basalt occurring at $80 \mathrm{~cm}$, where a chill zone had developed.

\section{LEG 59}

\section{Site 447 (see Fig. 7)}

The sedimentary sequence at this site (two holes, samples from Hole 447A alone were analyzed) has been divided into seven units. Unit $I$ is a pelagic brown clay and consists of abundant clay minerals and minor quartz and feldspar. Phillipsite has been found here as well as in the following samples from Units II, III, IV, and VII. Sample 5-2, 104-106 cm of Unit II is a late Oligocene calcareous pelagic clay and has, accordingly, 14.1 percent calcite. Only $\sim 6$ percent carbonate has been found in samples from Units III and IV, which were described as nannofossil chalk breccia and microcrystalline chalk breccia with clasts of altered pumice and basalt. Chlorite and kaolinite are present in small amounts only, whereas smectite becomes the dominant clay mineral and is the only one detectable in Sample $11-1,0-4 \mathrm{~cm}$ from a middle Oligocene volcaniclastic breccia (Unit VII). The bulk mineralogy of Sample 11-1, $0-4 \mathrm{~cm}$ is very similar to that of the two previous samples from Units III and IV.

\section{Site 448 (see Fig. 7)}

The sedimentary sequence at this site comprises five units. Unit I is a middle Miocene to late Oligocene nannofossil ooze with rare pumice fragments and traces of volcanic glass. All samples of Unit I are carbonate-rich, except for Sample 448-11-6, 58-60 cm. The clay mineral suite is dominated by smectite; only samples near the top of Unit I contain illite and/or kaolinite (+ chlorite). Quartz could not be detected in most samples, but is present in minor amounts throughout Holes 448 and 448A.

Unit II-late Oligocene-is of similar lithology but more indurated. Accordingly, samples that are less carbonate-rich have higher contents of volcanogenic mate- rial. Sample 20-3, 27-28 cm (Unit III) is a vitric tuff with some calcite, feldspar, quartz, chlorite, and hornblende. Unit IV is a middle Oligocene greenish and dark gray vitric tuff. All Unit IV samples analyzed have a similar composition with carbonate present in small or trace amounts and smectite as the dominant clay mineral. X-ray amorphous material is present in most samples. Volcanic glass has been altered to clay mineralsmainly smectite. Smectite is the main constituent of Sample 26-31, 71-74 cm.

Two samples of Unit V-middle Oligocene-interbedded with chalk and vitric tuff have been X-rayed and yielded low to medium feldspar contents and phillipsite, in addition to calcite (Sample 34-1, 29-31 cm). The Unit I samples of Hole 448A have high carbonate contents $(\sim 80 \%)$, whereas the third-a vitric tuff-is high in volcanic glass and has minor admixtures of quartz and feldspar.

\section{Site 449 (see Fig. 8)}

Five sedimentary units, ranging from Pleistocene to earliest Miocene were identified at this site. Unit I consists of Pleistocene to Miocene dark brown, pelagic clay. Quartz decreases from $\sim 12$ to $\sim 1$ percent downhole; illite is the main clay mineral on top, but could not be detected in the remaining samples. Sample 7-5, 81-83 cm (Unit III) is from a sequence of radiolarian-rich pelagic clays and nannofossil ooze; $\sim 5$ percent carbonate and 9 percent feldspar are present; the main constituent is $\mathrm{X}$-ray amorphous material.

Unit IV is a lower Miocene pelagic clay containing pumice fragments and volcanic glass. Carbonate is, according to shipboard and our own analyses, present in moderate amounts. Both of our samples contain phillipsite. Sample 13-3, 44-46 cm of Unit V is a nannofossil ooze with $\mathrm{CaCO}_{3}$ as the main constituent. Again phillipsite could be detected.

\section{Site 450 (see Fig. 9)}

At this site-also located in the Parece-Vela Basin333 meters of sediments, which overlie an intrusive basalt, have been drilled (see Fig. 1). Two major sedimentary units could be distinguished and are consequently divided into several sub-units. Unit $I$ is composed of pelagic clays with or without volcanic ash (Sub-units IA and IB). Samples from Sub-unit IA are characterized by moderate quartz and feldspar and relatively high illite contents. The latter mineral is lacking in Sub-unit IB and all deeper sections of Site 450. In Sub-unit IB we found $\sim 4$ to $\sim 8$ percent carbonate; in the glass-rich sample (Sample 4-2, 74-76 cm), no clay minerals could be detected. Volcanic glass is also an important constituent of the subsequent samples from Sub-units IIA and IIB, which are composed of middle Oligocene vitric tuffs and tuffaceous volcaniclastic conglomerates. The clay mineral suite is dominated by smectite, which is the only one detectable below Core 14. All samples contain moderate amounts of feldspar and calcite. Sample 36-1, $148-150 \mathrm{~cm}$ is from a hydrothermally altered fine vitric tuff (Sub-unit IIB); smectite is present and dominant and well crystallized. 


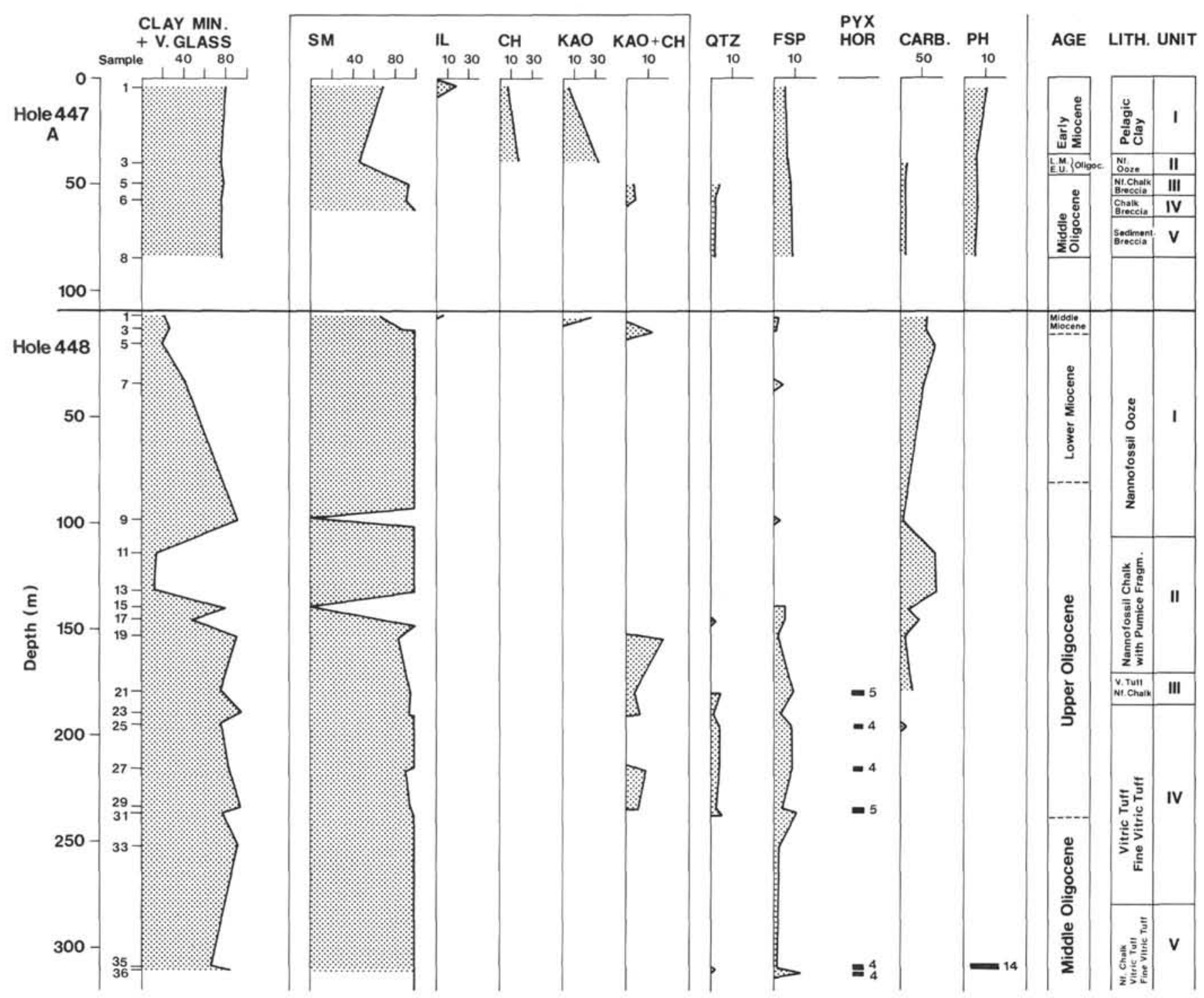

Figure 7. Hole 447A and 448: Semiquantitative determination of the mineral composition plotted versus depth below sea floor and lithology. Abbreviations as in Figure 2.

\section{Site 451 (see Fig. 10)}

At Site 451-West Mariana Ridge-three major sedimentary units were drilled. Unit I-early Pliocene to late Quaternary-consists of foraminiferal and nannofossiliferous oozes and is correspondingly rich in $\mathrm{CaCO}_{3}$. Here we found no clay, glass minerals, and only minor amounts of silicates. Some amorphous material (volcanic glass) is present. Unit II is a late Miocene to early Pliocene carbonate-rich vitric ash $(+$ minor amounts of vitric tuff). Calcite and feldspar are important constituents; volcanic glass and amorphous material are the main components of the analyzed samples.

Unit III-also late Miocene-is a sedimentary sequence of volcaniclastic material. It has been divided into seven sub-units. Sub-unit IIIA is a vitric tuff, vitric ash, and carbonate-rich vitric ash. Our samples have relatively high carbonate contents and moderate feldspar contents. In vitric tuffs and volcaniclastic breccias of Sub-unit IIIB, we found up to $\sim 49$ percent $\mathrm{CaCO}_{3}$; this reflects admixtures of organic carbonate (mainly foraminifers); some samples contain pyroxene. The sample from Sub-unit IIIC, a boulder volcaniclastic breccia grading to carbonate-rich vitric tuff, has moderate contents of $\mathrm{CaCO}_{3}$ and feldspar; minor amounts of pyroxene, hornblende and quartz are also present. No samples of Sub-unit IIID-black vitric tuffs-have been received. Sub-unit IIIE is interbedded with vitric tuff and volcaniclastic breccia. Here we found moderate to high feldspar contents (3.4 to 24\%) and traces of clinoptilolite (Sample 58-2, 53-55 $\mathrm{cm}$ ) and 6 percent analcime (Sample 62-2, 57-59 cm). Quartz could not be detected below Core 52, and pyroxene in samples below Core 62 . Sub-unit IIIF-vitric, lithic, and crystalline tuff, volcaniclastic conglomerate and breccia-has a similar mineralogical composition; one sample (Sample 74-2, 24-26 cm) contains 8 percent analcime. Traces of clinoptilolite have been found in most samples of Sub-unit 


\section{Hole 449}
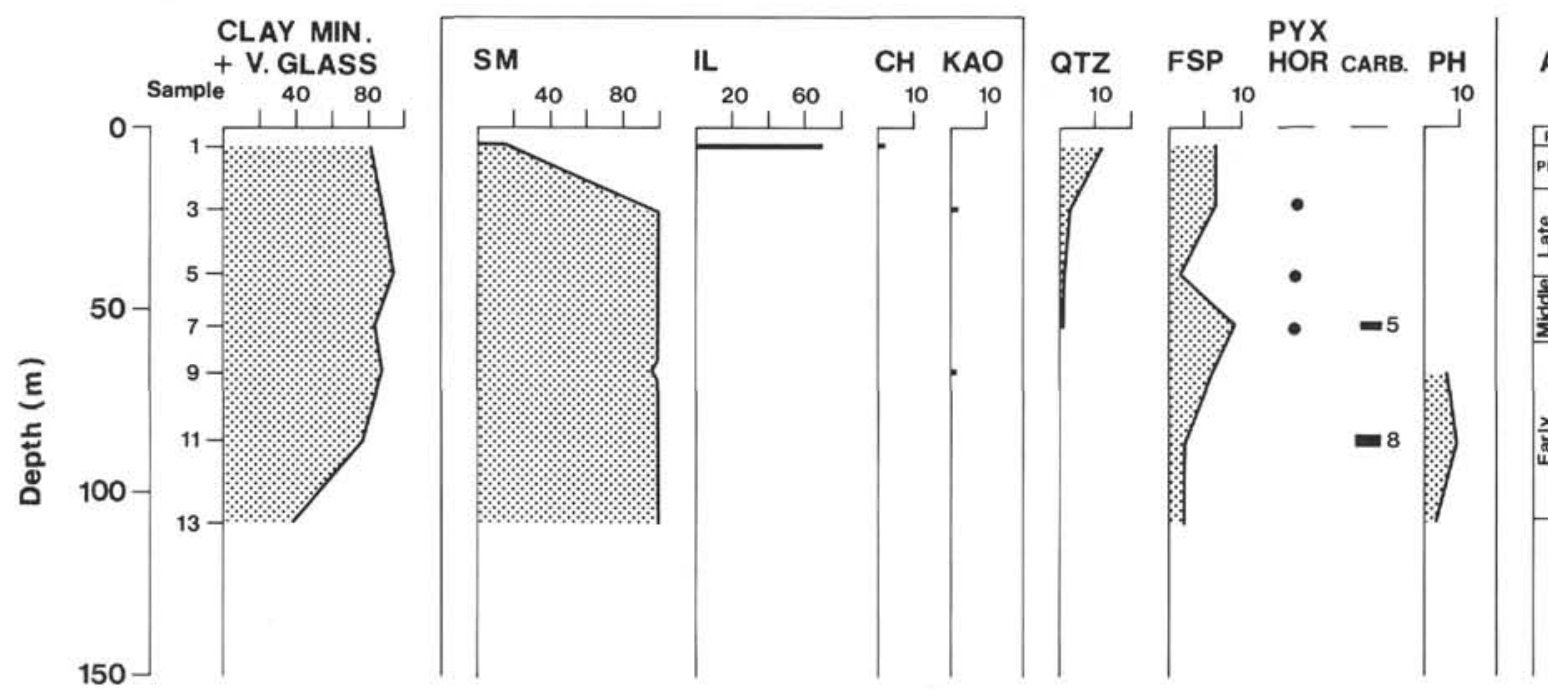

AGE

LITH. UNIT

Figure 8. Hole 449: Semiquantitative determination of the mineral composition plotted versus depth below sea floor and lithology. Abbreviations as in Figure 2.

IIIG-interbedded vitric tuff, volcaniclastic breccia and conglomerate. The main constituent, as in most previous samples, is volcanic glass; moderate feldspar contents prevail throughout the samples.

Smectite was the only clay mineral found in Hole 451 , except for Sample 100-1, 148-149 cm where $\sim 20$ percent illite has been detected. This sample comes from a volcaniclastic conglomerate which contains some pieces of hydrothermally altered andesite.

\section{LEG 60}

\section{Site 453 (see Fig. 11)}

Sediments encountered at this site, the westernmost of the three drilling targets in the Mariana Trough, consist of turbiditic muds, silts, and sands of volcanic debris (Unit I). Unit II is a polymict breccia of gabbro, diabase, and basalt fragments and sheared serpentinized gabbro. Smectite predominates the mineralogy of Unit I; only three samples contain less than 80 percent, and all others at least 90 percent. Two low-smectite samples have higher percentages of kaolinite and/or illite. The latter mineral as well as chlorite could not be detected in most of the samples.

Volcanogenic material, especially volcanic glass, is abundant throughout Hole 453, and pyroxene and hornblende have been found in most samples. Some Pliocene samples contain zeolites in percentages varying between 2 and 7 percent. In Sample 453-10-2, 89-92 cm (clay), 4 percent $\mathrm{CaCO}_{3}$ has been detected. All other samples were essentially carbonate-free, thus confirming shipboard results that deposition occurred at water depths close to the carbonate compensation depth.

\section{Site 454 (Hole 454A) (see Fig. 11)}

This site is located in the central Mariana Trough. Two holes had to be drilled because of adverse weather conditions during drilling operations. The sedimentary sequence of this turbiditic deposit has been divided into two units. Unit I is 67 meters thick and consists of late Pleistocene to early Pleistocene hemipelagic and biogenic (mainly siliceous) sediments. Unit II is composed of interbedded sediments with rare biogenic (mainly calcareous) components and basalts. Two vitric mud samples and one vitric ash sample of Unit I have been $\mathrm{X}$-rayed, their mineralogy being dominated by X-ray amorphous material, whereas smectite is the clay mineral present in higher amounts. Minor components include quartz, feldspar, chlorite, and kaolinite, in addition to pyroxene and hornblende. Sample 454A-2-2, 20-24 cm (vitric ash) does not contain quartz and has the least smectite content, whereas X-ray amorphous material is present in higher amounts.

\section{Site 455 (no sample received)}

\section{Site 456 (Hole 456A) (see Fig. 11)}

At this site-located in a small sediment pond on a local bathymetric high-again two holes had to be drilled. Hole 456 reached a depth of 168 meters but had to be abandoned because of drilling difficulties. Hole $456 \mathrm{~A}$ reached a depth of 159 meters and was cored to a depth of 140 meters. Three units have been distinguished. Unit I consists of Pleistocene vitric mud and marly nannofossil ooze with silty and sandy ash layers. Unit II-early Pleistocene-is a semilithified, partly bioturbated vitric mudstone, and vitric nannofossil chalk. Laminated graded vitric tuff (turbidite) is also present. Unit III is almost entirely basalt.

Four samples of Unit I have been X-rayed and analyzed for their carbonate contents. Only Unit I samples of Hole 456 contain $\mathrm{CaCO}_{3}$ in measurable quantities; those of Hole 456A are carbonate-free. Smectite and $\mathrm{X}$-ray amorphous material are the main constituents of all Unit I samples, whereas quartz, chlorite, and kao- 
Hole 450
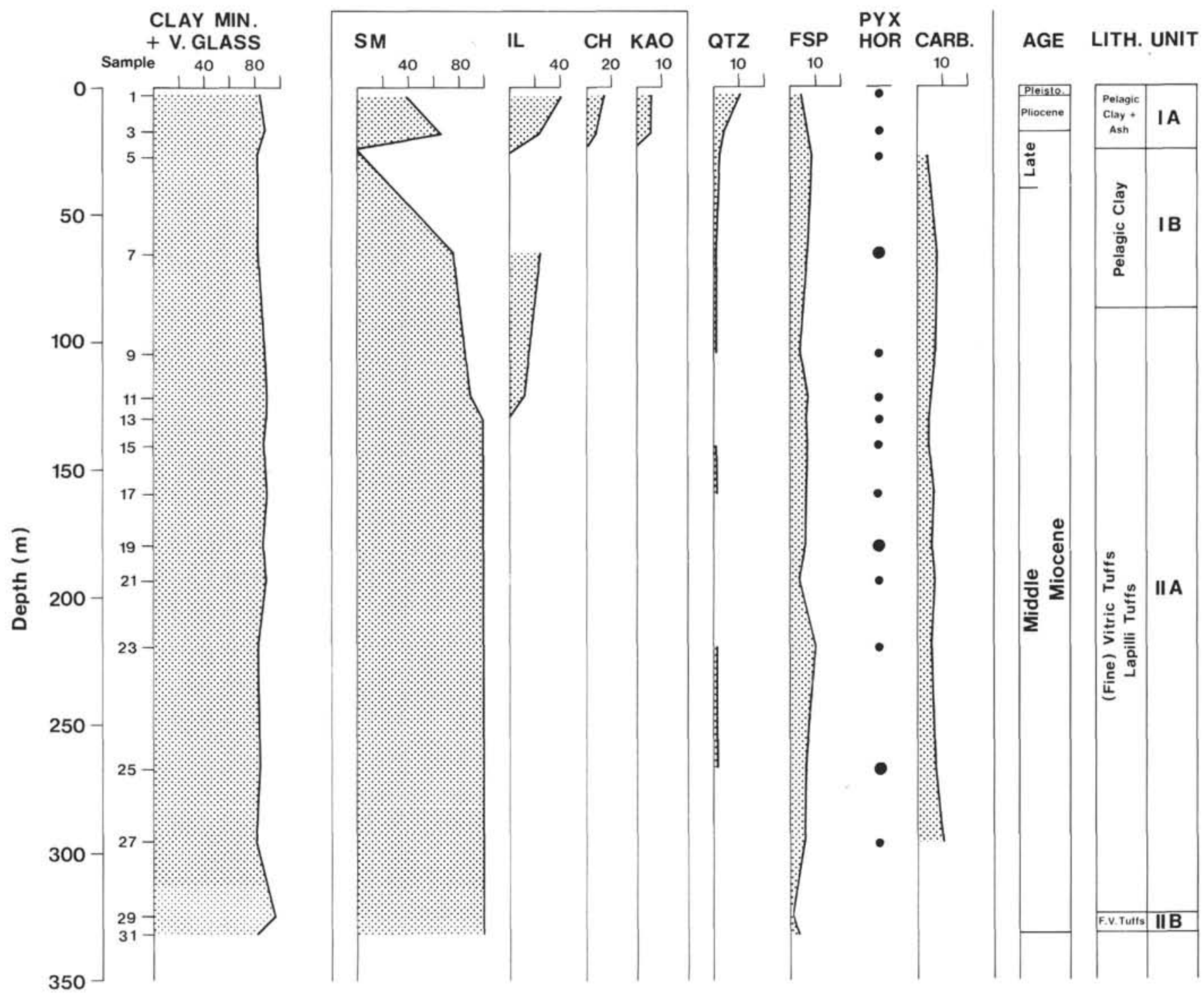

Figure 9. Hole 450: Semiquantitative determination of the mineral composition plotted versus depth below sea floor and lithology. Abbreviations as in Figure 2.

linite are present in minor amounts. Feldspar contents vary between 2,4 , and 8.4 percent.

The Unit II sample of Hole 456 yielded a high amount of volcanic glass. Other important constituents of the bulk sample mineralogy are quartz $(17.5 \%)$ and $\mathrm{CaCO}_{3}(13 \%)$. The clay mineral suite is dominated by kaolinite. Neither chlorite nor illite seems to be present. Sample 456A-7-1, 54-56 cm of a transition zone between sandstone and vitric mudstone with fine silt layers contains 25 percent analcime and minor feldspar. The other main constituents are smectite and a swelling chlorite which predominate the $<2-\mu \mathrm{m}$ fraction.

\section{Site 458 (see Fig. 11)}

This site was located on a gravity high between the Mariana Trench slope and the Mariana arc. Four sedimentary units could be distinguished: Unit I, which is dated Pleistocene to late Pliocene, consists of abundant volcanic ash (glass and feldspar) and siliceous and calcareous (biogenic) components. One sample of this unit was analyzed; it has abundant glass, 10 percent feldspar, and minor smectite as the only clay mineral.

Unit II-late Pliocene to earliest Pliocene-is of similar lithology as Unit I, but Unit III is a partly vitricsiliceous nannofossil chalk with some vitric-crystal mudstone and thin, silty to sandy vitric tuff layers. The analyzed sample (Sample 458-11, 13-14 cm) comes from a clayey nannofossil chalk sequence, but contains only 16 percent $\mathrm{CaCO}_{3}$ and no clay minerals. The main constituent is volcanic glass $(69 \%)$, and feldspar and quartz are present, too.

\section{Site 459 (see Fig. 11)}

At this site, which is located immediately above the Mariana Trench Slope break, 691.5 meters of sediment and igneous rocks were drilled. The sediments have been 


\section{Hole 451}

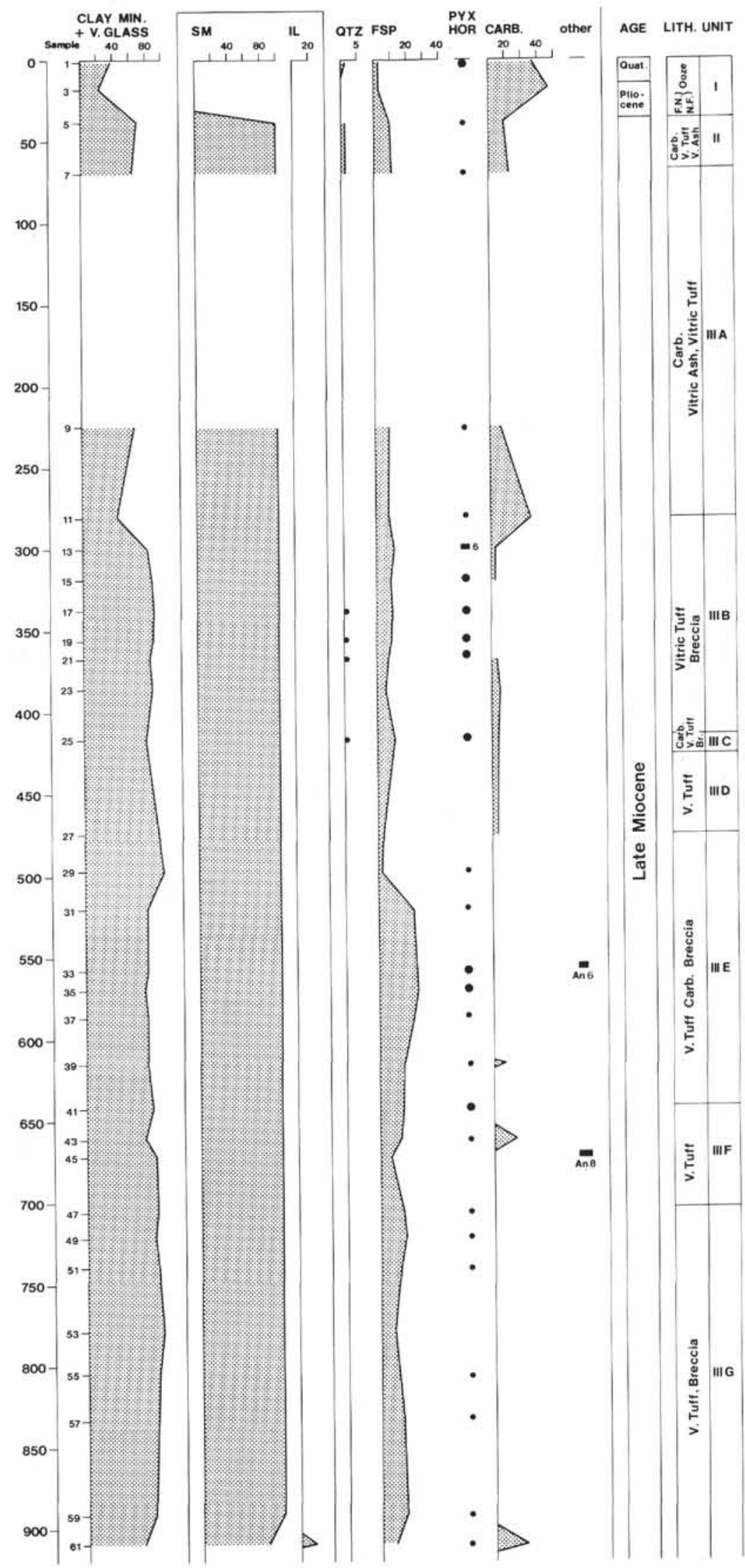

Figure 10. Hole 451: Semiquantitative determination of the mineral composition plotted versus depth below sea floor and lithology. Abbreviations as in Figure 2. 
U. NAGEL, G. MÜLLER, D. SCHUMANN

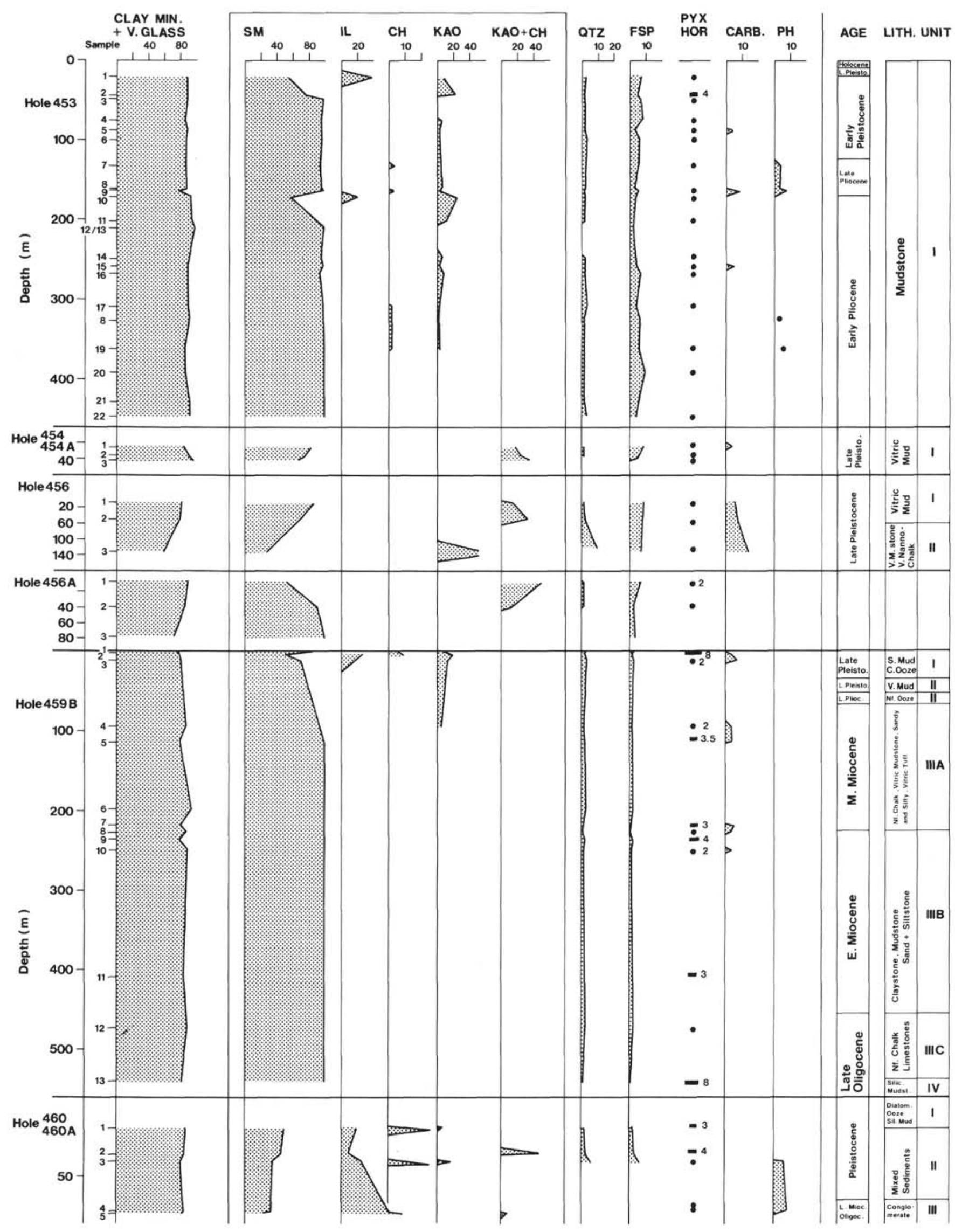

Figure 11. Holes 453, 454, 454A, 456, 456A, 459B, 460 and 460A:Semiquantitative determination of the mineral composition plotted versus depth below sea floor and lithology. Abbreviations as in Figure 2. 
divided into six units. Unit I-late Pleistocene-is represented by vitric siliceous and calcareous mud and ooze with some layering; three samples have been X-rayed. Their most notable feature is the decrease in feldspar content from 20 to 7.6 percent, whereas all other mineral percentages scatter. Smectite is present in all samples and results partly from the alteration of volcanogenic components. Illite was found in Unit I only.

No samples of Unit II have been received. Unit III is mainly turbiditic and has been divided into three subunits. Sub-unit IIIA is a middle Miocene turbiditic sequence of vitric marly nannofossil chalk, vitric mudstone, and sandy and silty vitric tuff. The first sample (Sample 459B-11-1, 80-82 cm) comes from a section which was described as "vitric marly nannofossil chalk," but the sample itself is described as a "drilling paste." Accordingly, it contains 85 percent amorphous material and clay minerals, of which smectite is the most prevalent one; minor admixtures include 4 percent $\mathrm{CaCO}_{3}, 7$ percent feldspar, and $\sim 1$ percent quartz.

Sample $13-1,32-34 \mathrm{~cm}$ is from a silty section of a vitric marly chalk with turbidite, but has only 3 percent $\mathrm{CaCO}_{3}$. From this section downward, smectite is the only clay mineral present. The next sample (Sample $21-1,70-78 \mathrm{~cm}$ ), from a similar sequence, is a mudstone, with smectite and amorphous material being the main constituents. Only minor amounts of quartz and feldspar and no $\mathrm{CaCO}_{3}$ are present. $\mathrm{CaCO}_{3}$ is a minor component of Sample 24-2, 84-86 cm, which is also from a turbiditic sequence.

Sub-unit IIIB-early Miocene-comprises again turbiditic sequences, which consist of claystones, mudstones, siltstones, and sandstones. Four samples of this sub-unit (three vitric mudstones, one from a contact of vitric mudstone and vitric tuff) have been $\mathrm{X}$-rayed and yield high amounts of volcanic glass and smectite. Quartz contents vary between 1 and 2 percent, feldspar between 4.6 and 13 percent. Carbonate contents are not high (0-3.8\%). Sample 44-2, 4-7 cm (contact) of vitric mudstone (vitric tuff) contains well-crystallized smectite.

Sub-unit IIIC was deposited during late Oligocene. This sequence is dominated by vitric nannofossil chalks. In Sample 51-1, 65-67 cm (from a vitric marly nannofossil chalk), however, no $\mathrm{CaCO}_{3}$ was found. Smectite is the most abundant mineral; admixtures include $\mathbf{2 . 2}$ percent quartz and 11 percent feldspar in addition to 1 percent pyroxene and traces of hornblende.

Unit IV is an early Oligocene claystone with vitric tuff layers. Sample $58-2,40-42 \mathrm{~cm}$ is similar to the Subunit IIIC sample described above, but pyroxene and hornblende are higher ( 3 and $4.5 \%$, respectively). No samples of Unit V and VI (claystones and silicified claystones) have been received.

\section{Site 460 (see Fig. 11)}

This site is located on the inner (arc-side) wall of the Mariana Trench. Two holes have been drilled; in each hole about 75 meters of sediments were penetrated. One sample of Hole 460 from a unit of undetermined age (but below Pleistocene), containing moderate brown crystals and with gray particles of diatomaceous ooze and a few thin ash layers, was X-rayed. 87 percent clay minerals ( + amorphous material) are present, of which smectite and chlorite are the most prevalent ones. Quartz and feldspar make up only 1.3 and 7.8 percent, respectively, of the bulk sample. Other components occur in insignificant amounts.

In Hole $460 \mathrm{~A}$ a strongly disturbed sequence was penetrated. Sample 460A-4-7, 18-20 cm is a late Pleistocene calcareous vitric mud and contains 3.2 percent $\mathrm{CaCO}_{3}$. Major components are smectite, chlorite, and kaolinite and amorphous material (including volcanic glass). Sample 5-3, 80-82 cm comes from a Quaternary vitric mud section and contains much volcanogenic material. Smectite, chlorite, and illite are present in similar amounts $(34.5,26.0$, and $26.3 \%$, respectively). 6 percent phillipsite, which probably resulted from the alteration of volcanic glass, has been identified in this sample. Minor admixtures include feldspar, quartz, and pyroxene, in addition to traces of hornblende.

Sample 8,CC has been dated as early Miocene or late Oligocene. Both samples are the matrix for altered basalts. Sample $8, C C, 21-23 \mathrm{~cm}$ is a conglomerate consisting of altered basalt fragments in a clayey matrix; here we again found phillipsite. Feldspar and phillipsite together comprise 16 percent of the bulk mineralogy, which is dominated by clay minerals. Minor amounts of pyroxene and hornblende (traces) are present as well. The sample from below $(33-35 \mathrm{~cm})$ consists only of feldspar, smectite, and illite, with illite-as in the previous sample-dominating. Only one sample has been received from Site 461 (Hole 461), on the inner wall of the Mariana Trench. It consisted of a Quaternary vitric, siliceous mud, rich in clay minerals.

\section{ACKNOWLEDGMENTS}

Financial support for these investigations was provided by the German Research Council (Deutsche Forschungsgemeinschaft). D. Godfrey and Ms. Beckenbach helped with the translations and typing of the manuscript.

\section{REFERENCES}

Mann, U., and Muller, G., 1980. X-Ray mineralogy of Deep Sea Drilling Project Legs 51 through 53, Western North Atlantic. In Donnelly, T., Francheteau, J., Bryan, W., Robinson, P., Flower, M., Salisbury, M., et al., Pt. 2, Init. Repts. DSDP, 51, 52, 53: Washington (U.S. Govt. Printing Office), 721-729.

Müller, G., and Gastner, M., 1971. The "Karbonat-Bombe," a simple device for the determination of the carbonate content in sediments, soils and other materials. N. Jb. Mineral. Mh., 10, 466-469. 